TRANSACTIONS OF THE

AMERICAN MATHEMATICAL SOCIETY

Volume 352, Number 1, Pages 95-114

$\mathrm{S}$ 0002-9947(99)02396-X

Article electronically published on March 8, 1999

\title{
TIGHT CLOSURE, PLUS CLOSURE AND FROBENIUS CLOSURE IN CUBICAL CONES
}

\author{
MOIRA A. MCDERMOTT
}

\begin{abstract}
We consider tight closure, plus closure and Frobenius closure in the rings $R=K[[x, y, z]] /\left(x^{3}+y^{3}+z^{3}\right)$, where $K$ is a field of characteristic $p$ and $p \neq 3$. We use a $\mathbb{Z}_{3}$-grading of these rings to reduce questions about ideals in the quotient rings to questions about ideals in the regular ring $K[[x, y]]$. We show that Frobenius closure is the same as tight closure in certain classes of ideals when $p \equiv 2 \bmod 3$. Since $I^{F} \subseteq I R^{+} \cap R \subseteq I^{*}$, we conclude that $I R^{+} \cap R=I^{*}$ for these ideals. Using injective modules over the ring $R^{\infty}$, the union of all $p^{e}$ th roots of elements of $R$, we reduce the question of whether $I^{F}=I^{*}$ for $\mathbb{Z}_{3}$-graded ideals to the case of $\mathbb{Z}_{3}$-graded irreducible modules. We classify the irreducible $m$-primary $\mathbb{Z}_{3}$-graded ideals. We then show that $I^{F}=$ $I^{*}$ for most irreducible $m$-primary $\mathbb{Z}_{3}$-graded ideals in $K[[x, y, z]] /\left(x^{3}+y^{3}+z^{3}\right)$, where $K$ is a field of characteristic $p$ and $p \equiv 2 \bmod 3$. Hence $I^{*}=I R^{+} \cap R$ for these ideals.
\end{abstract}

In this paper we discuss the conjecture that $I^{*}=I R^{+} \cap R$, where $R^{+}$denotes the integral closure of a domain $R$ of characteristic $p$ in an algebraic closure of its fraction field and $I^{*}$ denotes the tight closure of $I$. The ring $R^{+}$is characterized by the property that it is a domain integral over $R$ and every monic polynomial with coefficients in $R^{+}$factors into monic linear factors. This characterization can be used to prove the following property of $R^{+}$: If $W$ is a multiplicatively closed set of $R$, then $\left(W^{-1} R\right)^{+} \cong W^{-1} R^{+}$. Aside from providing a much more concrete description of tight closure, proving that $I^{*}=I R^{+} \cap R$ would solve the localization problem for tight closure. It is known that $I^{*}=I R^{+} \cap R$ for parameter ideals [Sm1] and for rings in which every ideal of the normalization is tightly closed. Also, for those ideals $I$ of an excellent local domain $R$ such that $R / I$ has finite phantom projective dimension, it is known that $I^{*}=I R^{+} \cap R[\mathrm{Ab}]$. However, the conjecture is open even for two-dimensional normal Gorenstein domains. In particular, the conjecture is open for the cubical cone $K[[x, y, z]] /\left(x^{3}+y^{3}+z^{3}\right)$, where $K$ is a field of characteristic $p$ and $p \neq 3$, and more generally for rings of the form $K[[x, y, z]] /(F(x, y, z))$ where $F$ is a homogeneous cubic polynomial.

We consider tight closure, plus closure and Frobenius closure in the rings $R=$ $K[[x, y, z]] /\left(x^{3}+y^{3}+z^{3}\right)$, where $K$ is a field of characteristic $p$ and $p \neq 3$. In Section 1 we use a $\mathbb{Z}_{3}$-grading of these rings to reduce questions about ideals in the quotient rings to questions about ideals in the regular rings $K[[x, y]]$. In Section 2 we show that the Frobenius closure of an ideal $I$, denoted $I^{F}$, is the same as the tight closure in certain classes of ideals when $p \equiv 2 \bmod 3$. Since $I^{F} \subseteq I R^{+} \cap R \subseteq I^{*}$,

Received by the editors August 27, 1997.

1991 Mathematics Subject Classification. Primary 13A35, 13A02, 13H10.

Key words and phrases. Tight closure, characteristic $p$, Frobenius closure, plus closure. 
we conclude that $I R^{+} \cap R=I^{*}$ for these ideals. In Section 3 we use injective modules over the ring $R^{\infty}$, the union of all $p^{e}$ th roots of elements of $R$, to reduce the question of whether $I^{F}=I^{*}$ for $\mathbb{Z}_{3}$-graded ideals to the case of $\mathbb{Z}_{3}$-graded irreducible modules. In Section 4 we classify the irreducible $m$-primary $\mathbb{Z}_{3}$-graded ideals and then show that $I^{F}=I^{*}$ for most irreducible $m$-primary $\mathbb{Z}_{3}$-graded ideals in $K[[x, y, z]] /\left(x^{3}+y^{3}+z^{3}\right)$, where $K$ is a field of characteristic $p$ and $p \equiv 2 \bmod 3$. Hence $I^{*}=I R^{+} \cap R$ for these ideals.

\section{Cubical Cones}

We denote by $\mathbb{Z}_{n}$ the ring $\mathbb{Z} / n \mathbb{Z}$. We first describe a $\mathbb{Z}_{3}$-grading on the cubical cones $R=K[[x, y, z]] /\left(x^{3}+y^{3}+z^{3}\right)$. We will also discuss tight closure and Frobenius closure in these rings before proving the main results, Theorem 2.1 and Theorem 4.5 .

$\mathbb{Z}_{3}$-grading. First we describe a $\mathbb{Z}_{n}$-grading of rings of the form $R=A[[z]] /\left(z^{n}-a\right)$ where $a \in A$. The ring $R$ has the following decomposition as an $A$-module: $R=$ $A \oplus A z \oplus \cdots \oplus A z^{n-1}$. Every element of $R$ can be uniquely expressed as an element of $A \oplus A z \oplus \cdots \oplus A z^{n-1}$ by replacing every occurrence of $z^{n}$ by $a$. $R$ is $\mathbb{Z}_{n}$-graded, where the $i$ th piece of $R$, denoted by $R_{i}$, is $A z^{i}, 0 \leq i<n$, since $A z^{i} A z^{j} \subseteq A z^{i+j}$ if $i+j<n$ and $A z^{i} A z^{j} \subseteq A z^{i+j-n}$ if $i+j \geq n$.

We use this idea to obtain a $\mathbb{Z}_{3}$-grading on $R=K[[x, y, z]] /\left(x^{3}+y^{3}+z^{3}\right)$ by letting $A=K[[x, y]]$. Let $H, I$, and $J$ be ideals of $K[[x, y]]$. Suppose $H \subseteq I \subseteq J \subseteq$ $H:\left(x^{3}+y^{3}\right)$. Then $H+I z+J z^{2}$ is an ideal of $R$. On the other hand, in order for a $\mathbb{Z}_{3}$-graded ideal to be closed under multiplication by $z$, it must have this form. Thus, it is easy to see that the ideals of $R$ homogeneous with respect to the $\mathbb{Z}_{3^{-}}$grading are precisely the ideals of this form. We can study the ideal $H+I z+J z^{2}$ by considering $(H, I, J)$, a triple of ideals in $K[[x, y]]$. Indeed, we will use the notation $(H, I, J)$ to denote the ideal $H+I z+J z^{2}$, and it is understood that $H, I$, and $J$ are ideals of $K[[x, y]]$. For example, the ideal $\left(x^{2}, y^{2} z, x z^{2}\right)$ is represented by the triple $(H, I, J)$ where $H=\left(x^{2}, y^{5}, x y^{3}\right), I=\left(x^{2}, y^{2}\right)$ and $J=\left(x, y^{2}\right)$.

If $R$ is a reduced ring of characteristic $p$, we write $R^{1 / q}$ for the ring obtained by adjoining $q$ th roots of all elements of $R$. Next we observe that the $\mathbb{Z}_{3}$-grading on $R$ extends to $R^{\infty}=\bigcup_{q} R^{1 / q}$. It is enough to show that the grading on $R$ extends to $R^{1 / q}$. If $u \in R_{i}$, then the image of $u$ is in $R_{j}^{1 / q}$ where $q i \equiv j \bmod 3$.

We now show that if $I$ is a graded ideal, then so is $I^{*}$.

(1.1) Lemma. Let $R$ be a finitely generated $k$-algebra that is $\mathbb{Z}_{n}$-graded and of characteristic $p$, where $p$ is not a prime factor of $n(p=0$ is allowed). Then the tight closure of a homogeneous ideal of $R$ is homogeneous.

Proof. Without loss of generality, we can assume $R$ is reduced, since the tight closure of $I$ is the preimage of the tight closure of the image of $I$ modulo the nilradical. Because the singular locus of $R$ is defined by a homogeneous ideal not contained in any minimal prime, $R$ has a homogeneous test element, say $c$. Let $I$ be a homogeneous ideal, and suppose that $z=z_{0}+z_{1}+\cdots+z_{n-1}$ is in $I^{*}$, where $z_{i}$ is the homogeneous component of $z$ of degree $i \bmod n$. Now we have $c z^{q}=c z_{0}^{q}+c z_{1}^{q}+\cdots+c z_{n-1}^{q}$ is in the homogeneous ideal $I^{[q]}$, and hence each of its homogeneous components is in $I^{[q]}$. But each of the elements $c z_{i}^{q}$ is homogeneous of degree $q i+\operatorname{deg} c \bmod n$, and since $q$ is invertible in $\mathbb{Z}_{n}$, these all have distinct 
degrees. Thus each $c z_{i}^{q} \in I^{[q]}$ for all $q \gg 0$ and each $z_{i} \in I^{*}$. This shows that $I^{*}$ is homogeneous.

Tight Closure and Frobenius Closure. We review the definition of tight closure for ideals of rings of characteristic $p>0$. Tight closure is defined more generally for modules and also for rings containing fields of arbitrary characteristic. See [HH1] or $[\mathrm{Hu}]$ for more details.

(1.2) Definition. Let $R$ be a ring of characteristic $p$ and $I$ be an ideal in a Noetherian ring $R$ of characteristic $p>0$. An element $u \in R$ is in the tight closure of $I$, denoted $I^{*}$, if there exists an element $c \in R$, not in any minimal prime of $R$, such that for all large $q=p^{e}, c x^{q} \in I^{[q]}$ where $I^{[q]}$ is the ideal generated by the $q$ th powers of all elements of $I$.

We denote by $I^{F}$ the Frobenius closure of an ideal $I$. Recall that $I^{F}=\{u \in$ $R: u^{q} \in I^{[q]}$ for some $\left.q\right\}$. We can also think of $I^{F}$ as $I R^{\infty} \cap R$, so $I^{F} \subseteq I R^{+} \cap R$, since $R^{\infty} \subseteq R^{+}$. In addition, we know that $I R^{+} \cap R \subseteq I^{*}[\mathrm{HH} 2]$. Hence $I^{F} \subseteq$ $I R^{+} \cap R \subseteq I^{*}$. So, if $I^{F}=I^{*}$, then that implies that $I^{*}=I R^{+} \cap R$.

An interesting bifurcation of this question in $R=K[[x, y, z]] /\left(x^{3}+y^{3}+z^{3}\right)$ depends on the characteristic of $K$. If $K$ has characteristic $p$ and $p \equiv 1 \bmod 3$, then $R$ is F-pure [HR, Proposition 5.21(c)] and $I^{F}=I$. We know that $I^{*} \neq I$ for some ideals of $R$, so $I^{F}$ cannot equal $I^{*}$, although it is still possible that $I^{*}=I R^{+} \cap R$. If $p \equiv 2 \bmod 3$, then $R$ is not $\mathrm{F}$-pure and it is conjectured that $I^{F}=I^{*}$ and hence that $I^{*}=I R^{+} \cap R$.

The goal of this paper is to show that $I^{*}=I^{F}$, and hence $I^{*}=I R^{+} \cap R$, for many graded ideals of $R$ when the characteristic of $K$ is congruent to $2 \bmod 3$.

Test Elements in Cubical Cones. In many applications one would like to be able to choose the element $c$ in the definition of tight closure independent of $x$ or $I$. It is very useful when a single choice of $c$, a test element, can be used for all tight closure tests in a given ring.

(1.3) Definition. The ideal of all $c \in R$ such that, for any ideal $I \subseteq R$, we have $c u^{q} \in I^{[q]}$ for all $q$ whenever $u \in I^{*}$ is called the test ideal for $R$. An element of the test ideal that is not in any minimal prime is called a test element. The ideal of all $c \in R$ such that for all parameter ideals (ideals generated by $i$ elements with height at least $i) I \subseteq R$, we have $c u^{q} \in I^{[q]}$ for all $q$ whenever $u \in I^{*}$ is called the parameter test ideal for $R$.

We now determine the test ideal for $K[[x, y, z]] /\left(x^{3}+y^{3}+z^{3}\right)$. The following proposition is proved for char $K \neq 2,3$ using a somewhat different method in [Sm2].

(1.4) Proposition. Let $R=K[[x, y, z]] /\left(x^{3}+y^{3}+z^{3}\right)$, where $K$ is a field of characteristic $p$ and $p \neq 3$. Then the maximal ideal, $m$, is the test ideal.

Proof. First note that we can reduce to the case where $K$ is algebraically closed. Enlarging $K$ to an algebraic closure is an integral extension and will not affect tight closure.

Let $\tau$ be the parameter test ideal for $R$. By Proposition 4.4(iii) of [Sm2], we know that $\tau=\left\{c \in R\right.$ such that $c\left(x^{t}, y^{t}\right)^{*} \subset\left(x^{t}, y^{t}\right)$ all $\left.t \in \mathbb{N}\right\}$. Since $R$ is Gorenstein, the test ideal is the same as the parameter test ideal [Sm2, Proposition 4.4].

We will show that $\left(x^{t}, y^{t}\right)^{*}=\left(x^{t}, y^{t}, x^{t-1} y^{t-1} z^{2}\right)$. Then it is clear that $\tau=$ $(x, y, z)$ since $\left(x^{t}, y^{t}\right):\left(x^{t}, y^{t}, x^{t-1} y^{t-1} z^{2}\right)=(x, y, z)$. Let $I=\left(x^{t}, y^{t}\right)$ and $J=$ 
$\left(x^{t}, y^{t}, x^{t-1} y^{t-1} z^{2}\right)$. The socle $\bmod J$ is generated by $u_{1}=x^{t-2} y^{t-1} z^{2}, u_{2}=$ $x^{t-1} y^{t-2} z^{2}$ and $u_{3}=x^{t-1} y^{t-1} z$. To see that $I^{*}=J$, it suffices to show that $\sum K u_{i} \cap I^{*}=0$, since if $J \subsetneq I^{*}$, then $I^{*}$ has nonzero intersection with $J: m$.

We would like to see that $\lambda_{1} u_{1}+\lambda_{2} u_{2}+\lambda_{3} \notin\left(x^{t}, y^{t}\right)^{*}$ where $\lambda_{i} \in K$. Using the $\mathbb{Z}_{3}$-grading, it is enough to show that $\lambda_{3} u_{3} \notin\left(x^{t}, y^{t}\right)^{*}$ and $\lambda_{1} u_{1}+\lambda_{2} u_{2} \notin\left(x^{t}, y^{t}\right)^{*}$. Using the $\mathbb{Z}_{3}$-grading again, but now letting $x$ play the role of $z\left(R=A[[x]] /\left(x^{3}-a\right)\right.$, $A=K[[y, z]])$, we can reduce the problem to showing $\lambda_{1} u_{1} \notin\left(x^{t}, y^{t}\right)^{*}, \lambda_{2} u_{2} \notin$ $\left(x^{t}, y^{t}\right)^{*}$ and $\lambda_{3} u_{3} \notin\left(x^{t}, y^{t}\right)^{*}$.

Suppose $u_{3} \in\left(x^{t}, y^{t}\right)^{*}$. Then $z \in\left(x^{t}, y^{t}\right)^{*}: x^{t-1} y^{t-1}$. We claim that $\left(x^{t}, y^{t}\right)^{*}: x^{t-1} y^{t-1} \subseteq(x, y)^{*}$. Let $u \in\left(x^{t}, y^{t}\right)^{*}: x^{t-1} y^{t-1}$, so $u x^{t-1} y^{t-1} \in\left(x^{t}, y^{t}\right)^{*}$. Then there exists $c$ such that $c u^{q} x^{(t-1) q} y^{(t-1) q} \in\left(x^{t q}, y^{t q}\right)$. This implies that $c u^{q} \in\left(x^{t q}, y^{t q}\right): x^{(t-1) q} y^{(t-1) q}$. But $\left(x^{t q}, y^{t q}\right): x^{(t-1) q} y^{(t-1) q} \subseteq\left(x^{q}, y^{q}\right)^{*}$ by a colon capturing argument [HH1, Theorem 7.15a]. So $c u^{q} \in\left(x^{q}, y^{q}\right)^{*}$, and we can find a test element $d$ such that $d c u^{q} \in\left(x^{q}, y^{q}\right)$ for all $q$. In other words, $u \in(x, y)^{*}$. Thus $x^{t-1} y^{t-1} z \in\left(x^{t}, y^{t}\right)^{*}$ implies $z \in(x, y)^{*}$, but we know that $z \notin(x, y)^{*}$ by a degree argument [Sm3, Theorem 2.2].

Now suppose $u_{1} \in\left(x^{t}, y^{t}\right)^{*}$. This implies that $z^{2} \in\left(x^{t}, y^{t}\right)^{*}: x^{t-2} y^{t-1}$. Using the same argument as before, we can show that $\left(x^{t}, y^{t}\right)^{*}: x^{t-2} y^{t-1} \subseteq\left(x^{2}, y\right)^{*}$. By symmetry, we must also have $z^{2} \in\left(x, y^{2}\right)^{*}$. So $z^{2} \in\left(x^{2}, y\right)^{*} \cap\left(x, y^{2}\right)^{*}$ which is contained in $\left(x^{2}, x y, y^{2}\right)^{*}$ by Theorem 7.12 of [HH1]. Again, $z^{2} \notin\left(x^{2}, x y, y^{2}\right)^{*}$ by degree arguments [Sm3, Theorem 2.2].

The fact that $m$ is the test ideal provides quite a lot of information. For example, using the fact that $m$ is the test ideal, we may conclude that if $u \in I^{*} \backslash I$, then $u$ is in the socle $\bmod I$.

(1.5) Proposition. Let $(R, m)$ be a local ring. Suppose $m$ is the test ideal. If $u \in I^{*} \backslash I$, then $u$ is in the socle $\bmod I$.

Proof. Let $u \in I^{*} \backslash I$. Then $m u^{q} \subseteq I^{[q]}$ for all $q$. In particular, $m u \subseteq I$. This says exactly that $u$ is in the socle $\bmod I$.

(1.6) Remark. Although determining whether an element is in the tight closure or Frobenius closure of an ideal involves checking certain conditions for infinitely many values of $q=p^{e}$, there are some instances where one $q$ is enough. If $c$ is a test element and $c u^{q} \notin I^{[q]}$ for some $q$, then $u \notin I^{*}$. Similarly, if $u^{q} \in I^{[q]}$ for some $q$, then $u^{q^{\prime}} \in I^{\left[q^{\prime}\right]}$ for all $q^{\prime} \geq q$ and hence $u \in I^{F}$.

In either situation, since we only need one $q$ that works, we can pick whichever value of $q$ is most helpful. For example, when $p \equiv 2 \bmod 3, p^{2 e} \equiv 1 \bmod 3$ and $p^{2 e+1} \equiv 2 \bmod 3$. It is often easier to work with powers of $p$ with a particular residue mod 3 and so we may choose $q$ accordingly.

Applications of the $\mathbb{Z}_{3}$-grading to Tight Closure. When trying to determine $I^{*}$ and $I^{F}$ for a given ideal $I$, we are interested in calculating $I^{[q]}$ and $I: m$. We will first calculate $I: m$.

(1.7) Lemma. Let $R=K[[x, y, z]] /\left(x^{3}+y^{3}+z^{3}\right)$, and let $H+I z+J z^{2}$ be $a$ $\mathbb{Z}_{3}$-graded ideal in $R$. Then $\left(H+I z+J z^{2}\right):(x, y, z)=$

$$
((H:(x, y)) \cap I)+((I:(x, y)) \cap J) z+\left((J:(x, y)) \cap\left(H:\left(x^{3}+y^{3}\right)\right)\right) z^{2} .
$$

Proof. Let $R_{i}$ denote the $i \bmod 3$ graded piece of $R$. Suppose $r \in R_{0}$ and $r \in$ $\left(H+I z+J z^{2}\right):(x, y, z)$. So we must have $r(x, y) \subseteq H$ and $r z \in I z$. In other words, 
$r \in(H:(x, y)) \cap I$. Similarly, if $r z \in R_{1}$ and $r z \in\left(H+I z+J z^{2}\right):(x, y, z)$, we must have $r \in(I:(x, y)) \cap J$. Let $r z^{2} \in R_{2}$ and suppose $r \in\left(H+I z+J z^{2}\right):(x, y, z)$. Again, we see that $r \in J:(x, y)$. We also know that $\left(r z^{2}\right) z=r\left(x^{3}+y^{3}\right) \in$ $\left(H+I z+J z^{2}\right)$. Since $r\left(x^{3}+y^{3}\right) \in R_{0}$, we must have $r\left(x^{3}+y^{3}\right) \in H$. In other words, $r \in H:\left(x^{3}+y^{3}\right)$. So $r \in\left((J:(x, y)) \cap\left(H:\left(x^{3}+y^{3}\right)\right)\right)$.

Next we will determine $I^{[q]}$ when $q \equiv 2 \bmod 3$.

(1.8) Lemma. Let $R=K[[x, y, z]] /\left(x^{3}+y^{3}+z^{3}\right)$, where $K$ is a field of characteristic $p$ and $p \equiv 2 \bmod 3$. Let $q=p^{2 e+1}=3 h+2$ and let $f=x^{3}+y^{3}$. Let $H+I z+J z^{2}$ be a $\mathbb{Z}_{3}$-graded ideal in $R$. Then

$$
\begin{aligned}
\left(H+I z+J z^{2}\right)^{[q]} & =\left(H^{[q]}+I^{[q]} f^{h+1}+J^{[q]} f^{2 h+2}\right) \\
& +\left(H^{[q]}+I^{[q]} f^{h+1}+J^{[q]} f^{2 h+1}\right) z+\left(H^{[q]}+I^{[q]} f^{h}+J^{[q]} f^{2 h+1}\right) z^{2} .
\end{aligned}
$$

Let $u=u_{0}+u_{1} z+u_{2} z^{2}$. Then $u^{q} \in\left(H+I z+J z^{2}\right)^{[q]}$ in $R$ if and only if

$$
\begin{aligned}
u_{0}{ }^{q} & \in\left(H^{[q]}+I^{[q]} f^{h+1}+J^{[q]} f^{2 h+2}\right), \\
u_{1}^{q} f^{h} & \in\left(H^{[q]}+I^{[q]} f^{h}+J^{[q]} f^{2 h+1}\right), \\
u_{2}^{q} f^{2 h+1} & \in\left(H^{[q]}+I^{[q]} f^{h+1}+J^{[q]} f^{2 h+1}\right) \quad \text { in } K[[x, y]] .
\end{aligned}
$$

Proof. We start by noting that $\left(H+I z+J z^{2}\right)^{[q]}$ is generated by $H^{[q]}+I^{[q]} z^{q}+J^{[q]} z^{2 q}$. Rewriting this using $q=3 h+2$ and the basic relation in $R, z^{3}=-\left(x^{3}+y^{3}\right)$, yields $H^{[q]}+I^{[q]} f^{h} z^{2}+J^{[q]} f^{2 h+1} z$. We will first consider $\left(H+I z+J z^{2}\right)^{[q]} \cap R_{0}$. If we multiply $I^{[q]} f^{h} z^{2}$ by $z$, we get $I^{[q]} f^{h} z^{3}=I^{[q]} f^{h+1}$ which is in $R_{0}$. Similarly, multiplying $J^{[q]} f^{2 h+1} z$ by $z^{2}$ gives $J^{[q]} f^{2 h+1} z^{3}=J^{[q]} f^{2 h+2}$. Thus,

$$
\left(H+I z+J z^{2}\right)^{[q]} \cap R_{0}=\left(H^{[q]}+I^{[q]} f^{h+1}+J^{[q]} f^{2 h+2}\right) .
$$

Similar arguments show that

$$
\begin{aligned}
& \left(H+I z+J z^{2}\right)^{[q]} \cap R_{1}=\left(H^{[q]}+I^{[q]} f^{h}+J^{[q]} f^{2 h+1}\right) \text { and } \\
& \left(H+I z+J z^{2}\right)^{[q]} \cap R_{2}=\left(H^{[q]}+I^{[q]} f^{h+1}+J^{[q]} f^{2 h+1}\right) .
\end{aligned}
$$

Since $u^{q}=u_{0}^{q}+u_{2} f^{2 h+1} z+u_{1} f^{2 h} z^{2}$, the last statement in the lemma is now clear.

Next we determine $I^{[q]}$ when $q \equiv 1 \bmod 3$.

(1.9) Lemma. Let $R=K[[x, y, z]] /\left(x^{3}+y^{3}+z^{3}\right)$, where $K$ is a field of characteristic $p$ and $p \equiv 2 \bmod 3$. Let $q=p^{2 e}=3 h+1$ and let $f=x^{3}+y^{3}$. Let $H+I z+J z^{2}$ be a $\mathbb{Z}_{3}$-graded ideal in $R$. Then

$$
\begin{aligned}
\left(H+I z+J z^{2}\right)^{[q]}= & \left(H^{[q]}+I^{[q]} f^{h+1}+J^{[q]} f^{2 h+1}\right) \\
& +\left(H^{[q]}+I^{[q]} f^{h}+J^{[q]} f^{2 h+1}\right) z+\left(H^{[q]}+I^{[q]} f^{h}+J^{[q]} f^{2 h}\right) z^{2} .
\end{aligned}
$$

Let $u=u_{0}+u_{1} z+u_{2} z^{2}$. Then $u^{q} \in\left(H+I z+J z^{2}\right)^{[q]}$ in $R$ if and only if

$$
\begin{aligned}
u_{0}{ }^{q} & \in\left(H^{[q]}+I^{[q]} f^{h+1}+J^{[q]} f^{2 h+1}\right), \\
u_{1}{ }^{q} f^{h} & \in\left(H^{[q]}+I^{[q]} f^{h}+J^{[q]} f^{2 h+1}\right), \\
u_{2}^{q} f^{2 h} & \in\left(H^{[q]}+I^{[q]} f^{h}+J^{[q]} f^{2 h}\right) \quad \text { in } K[[x, y]] .
\end{aligned}
$$


Proof. The proof is identical to the proof of Lemma 1.8 except we use $q=3 h+1$.

Note that $f^{h}=\left(x^{3}+y^{3}\right)^{h}$ appears often in the calculations. The question of whether a given element is in the tight closure of an ideal often comes down to whether or not a certain power of $f$ is contained in $\left(x^{q}, y^{q}\right)$. To this end, we establish the following lemmas which will be useful in showing that $I^{*}=I^{F}$.

(1.10) Lemma. Let $A=K[[x, y]]$, where $K$ is a field of characteristic $p$ and $p \equiv 2 \bmod 3$. Let $p=3 h+2$ and let $f=x^{3}+y^{3}$. Then $f^{2 h} \notin\left(x^{p}, y^{p}\right)$. Let $q=p^{2 e}=3 k+1 ;$ then $f^{2 k} \in\left(x^{q}, y^{q}\right)$.

Proof. Expand $f^{2 h}=\left(x^{3}+y^{3}\right)^{2 h}$ using the binomial theorem. Since $\left(\begin{array}{c}2 h \\ h\end{array}\right) x^{3 h} y^{3 h}$ is a term in the expansion and $x^{3 h} y^{3 h} \notin\left(x^{3 h+2}, y^{3 h+2}\right)=\left(x^{p}, y^{p}\right)$, it suffices to see that $\left(\begin{array}{c}2 h \\ h\end{array}\right) \not \equiv 0$ mod $p$. But $2 h<p$, so $p$ does not divide $\left(\begin{array}{c}2 h \\ h\end{array}\right)$.

As in the above case, $f^{2 k} \in\left(x^{q}, y^{q}\right)$ if and only if $\left(\begin{array}{c}2 k \\ k\end{array}\right) \equiv 0 \bmod p$. Suppose we know that $z^{2 q} \in\left(x^{q}, y^{q}\right) R$ where $q=3 k+1$. Using the basic relation in $R$ we see that $z^{2 q} \in\left(x^{q}, y^{q}\right) R$ if and only if $f^{2 k} z^{2} \in\left(x^{q}, y^{q}\right) R$. Using the $\mathbb{Z}_{3}$-grading we see that this is equivalent to having $f^{2 k} \in\left(x^{q}, y^{q}\right) A$. Expand $f^{2 k}$ using the binomial theorem to see that this is equivalent to having $\left(\begin{array}{c}2 k \\ k\end{array}\right) \equiv 0 \bmod p$. In other words, $\left(\begin{array}{c}2 k \\ k\end{array}\right) \equiv 0 \bmod p$ if and only if $z^{2 q} \in\left(x^{q}, y^{q}\right) R$ where $q=3 k+1$. We know that $z^{2 p} \in\left(x^{p}, y^{p}\right) R$ when $p \equiv 2 \bmod 3$ by the proof of Proposition 4.3. This implies that $z^{2 q} \in\left(x^{q}, y^{q}\right)$ for all $q=p^{e}$, in particular for $q=3 k+1$. Hence $\left(\begin{array}{c}2 k \\ k\end{array}\right) \equiv 0 \bmod p$, and $f^{2 k} \in\left(x^{q}, y^{q}\right)$.

We will use the following result about calculating binomial coefficients mod $p$ in Lemma 1.12 .

(1.11) Lucas's Theorem. Let $p$ be a prime and let $n=\sum_{0}^{s} a_{i} p^{i}, 0 \leq a_{j}<p$, $m=\sum_{0}^{s} b_{i} p_{i}, 0 \leq b_{k}<p$. Then $\left(\begin{array}{c}n \\ m\end{array}\right) \equiv\left(\begin{array}{l}a_{0} \\ b_{0}\end{array}\right)\left(\begin{array}{l}a_{1} \\ b_{1}\end{array}\right) \cdots\left(\begin{array}{l}a_{s} \\ b_{s}\end{array}\right) \bmod p$.

Proof. See [Fi, Theorem 1] or [L, p. 230].

(1.12) Lemma. Let $A=K[[x, y]]$, where $K$ be a field of characteristic $p$ and $p \equiv 2 \bmod 3$. Let $q=p^{2 e}=3 h+1$ and $f=x^{3}+y^{3}$. Then $\left(\begin{array}{c}3 h-2 \\ h-1\end{array}\right) \not \equiv 0 \bmod p$ and $f^{2 h-2} \in\left(x^{q}, y^{q}\right)$ except when $q=25$.

Proof. Since $p^{2 e}=3 h+1$, we can write $3 h-2=p^{2 e}-3$. So $\left(\begin{array}{c}3 h-2 \\ h-1\end{array}\right)=$ $\left(p^{2 e}-3\right)\left(p^{2 e}-4\right) \cdots\left(p^{2 e}-(h+1)\right) / 1 \cdot 2 \cdots(h-1)$. It is easy to show that $\left(\begin{array}{c}3 h-2 \\ h-1\end{array}\right)$ is divisible by $p$ if and only if $\left(\left(p^{2 e}-h\right)\left(p^{2 e}-(h+1)\right)\right) / 2$ is divisible by $p$. Routine divisibility arguments show that this cannot happen.

To see that $f^{2 h-2} \in\left(x^{q}, y^{q}\right)$, we expand $f^{2 h-2}$ using the binomial theorem. It is sufficient to show that $\left(\begin{array}{c}2 h-2 \\ h-1\end{array}\right)$ and $\left(\begin{array}{c}2 h-2 \\ h\end{array}\right)$ are congruent to zero $\bmod p$. If $p \neq 2$, then $p$ divides $\left(\begin{array}{c}2 h-2 \\ h\end{array}\right)$ if and only if $p$ divides $\left(\begin{array}{c}2 h-2 \\ h-1\end{array}\right)$. Next note that if $p \neq 2,5$, then $p$ divides $\left(\begin{array}{c}2 h \\ h\end{array}\right)$ if and only if $p$ divides $\left(\begin{array}{c}2 h-2 \\ h-1\end{array}\right)$.

We know from Proposition 1.10 that $p$ divides $\left(\begin{array}{c}2 h \\ h\end{array}\right)$ for the values of $h$ we are considering, so if $p \neq 2,5$, we know that $p$ also divides $\left(\begin{array}{c}2 h-2 \\ h-1\end{array}\right)$ and $\left(\begin{array}{c}2 h-2 \\ h\end{array}\right)$. If $p=2$, using (1.11), we can show that $\left(\begin{array}{c}2 h-2 \\ h\end{array}\right)=\left(\begin{array}{c}\text { even } \\ \text { odd }\end{array}\right) \equiv 0 \bmod 2$ and $\left(\begin{array}{c}2 h-2 \\ h-1\end{array}\right)=\left(\begin{array}{c}2 h-1) \\ h-1\end{array}\right) \equiv$ $0 \bmod 2$.

It remains to see that $\left(\begin{array}{c}2 h-2 \\ h\end{array}\right) \equiv 0 \bmod 5$ and $\left(\begin{array}{c}2 h-2 \\ h-1\end{array}\right) \equiv 0 \bmod 5$. We know from above that if $p \neq 2$, then it is enough to show that $\left(\begin{array}{c}2 h-2 \\ h\end{array}\right) \equiv 0 \bmod 5$. Write $5^{2 e}=3 h+1$. Using (1.11), we see that $\left(\begin{array}{c}2 h-2 \\ h\end{array}\right) \equiv 0 \bmod 5$ as long as $5^{2 e}>25$. 
At times, we will be able to make use of the fact that we are working over a regular ring or that $K[[x, y, z]] /\left(x^{3}+y^{3}+z^{3}\right)$ is flat as a $K[[x, y]]$-module. The following lemma and corollary provide useful information in these situations.

(1.13) Lemma. Let $R, S$ be arbitrary Noetherian rings such that $S$ is a flat $R$ algebra, and let $I, J$ be ideals of $R$. Then $I S:{ }_{S} J S=\left(I:{ }_{R} J\right) S$, where $I:{ }_{R} J=$ $\{r \in R: r J \subseteq I\}$.

Proof. See [N, Theorem 18.1, part 2].

(1.14) Corollary. In a regular ring $R$ of characteristic $p$, for any two ideals $I, J$ we have $I^{[q]}:{ }_{R} J^{[q]}=\left(I:{ }_{R} J\right)^{[q]}$ for all $q$. In particular, $I^{[q]}: x^{q}=(I: x)^{[q]}$ for all $q$.

Proof. See Corollary 4.3 of [HH1]. The statement follows from Lemma 1.13, since the iterated Frobenius endomorphism $F^{e}: R \rightarrow R$ is flat when $R$ is regular [K, Theorem 2.1] and $I^{[q]}=F^{e}(I) R$.

\section{Tight Closure and Frobenius Clonure in Cubical Cones}

We can now show that $I^{*}=I^{F}$ for some not necessarily irreducible ideals. We will discuss irreducible ideals in Section 4.

(2.1) Proposition. Let I be a $\mathbb{Z}_{3}$-graded ideal of $K[[x, y, z]] /\left(x^{3}+y^{3}+z^{3}\right)$, where $K$ is a field of characteristic $p$ and $p \equiv 2 \bmod 3$. Let $f=x^{3}+y^{3}$. If I has any of the following forms, then $I^{*}=I^{F}$.

(1) $(H, H, H)$,

(2) $(H, H, H:(x, y))$,

(3) $(H, H:(x, y), H: f)$,

(4) $(H, H:(x, y), H:(x, y))$,

(5) $\left(H, H, H:\left(x^{2}, y\right)\right)$.

In fact, in (2)-(5), $I$ is tightly closed, i.e. $I=I^{*}$.

Proof. We know that if $u \in I^{*} \backslash I$, then $u$ is in the socle mod $I$ (Proposition 1.5), so it is sufficient to check whether elements of the socle are in $I^{*}$ and $I^{F}$.

Proof of (1). Let $q=3 h+2$. Using the $\mathbb{Z}_{3}$-grading (Lemma 1.7) we know that $I:(x, y, z)=H+H z+(H:(x, y)) z^{2}$. So the socle $\bmod I$ is in $R_{2}$, the second graded piece of $R$. Let $u \in(H:(x, y)) \backslash H$. Then $u z^{2} \in R_{2}$ represents an element of the socle mod $I$. The test ideal is $(x, y, z)$ by Proposition 1.4. If $u z^{2} \in I^{*}$, then, using $z$ as a test element, and the grading (Lemma 1.8), we see that this is equivalent to having $u^{q} f^{2 h+1} \in H^{[q]}+H^{[q]} f^{h}+H^{[q]} f^{2 h+1}$ in $K[[x, y]]$, which implies that $u^{q} f^{2 h+1} \in H^{[q]}$. This, however, is exactly what is needed to have $\left(u z^{2}\right)^{q} \in I^{[q]}$ (Lemma 1.8) and hence $u z^{2} \in I^{F}$.

We can also show that $I^{*} \neq I$ in this case; in other words, $u z^{2}$ is always in $I^{*}$. In fact we can show that $u z^{2} \in I^{F}$. If $u z^{2} \in I^{F}$, then we must have $u^{q} z^{2 q} \in I^{[q]}$. This is equivalent to having $z^{2 q} \in I^{[q]}:{ }_{R} u^{q}$. Since $R$ is a flat $K[[x, y]]$-algebra, $I^{[q]}:{ }_{R} u^{q}=\left(I^{[q]}:{ }_{K[[x, y]]} u^{q}\right) R$ (Lemma 1.13). Since $K[[x, y]]$ is a regular local ring, $\left(I^{[q]}:_{K[[x, y]]} u^{q}\right) R=\left(I:_{K[[x, y]]} u\right)^{[q]} R$ (Corollary 1.14). Since $I$ is just the expansion of $H$ to $R$,

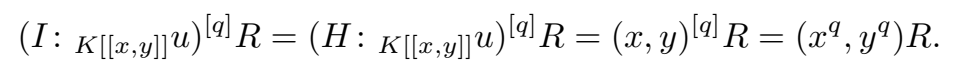


Thus, $u z^{2} \in I^{F}$ if and only if $z^{2 q} \in\left(x^{q}, y^{q}\right)$, which it is by the proof of Proposition 4.3 .

Proof of (2). Let $q=p^{2 e}=3 h+1$. Using the $\mathbb{Z}_{3}$-grading we know that $I:(x, y, z)=H+(H:(x, y)) z+\left(H:\left(x^{2}, x y, y^{2}\right)\right) z^{2}$ (Lemma 1.7). So the socle has components in $R_{1}$ and $R_{2}$. Let $u \in(H:(x, y)) \backslash H$. Then $u z$ represents an element of the socle mod $I$ and $u z$ is in $R_{1}$. If $u z \in I^{*}$, then, using $x$ as a test element and the grading (Lemma 1.9), we know that

$$
\begin{aligned}
x u^{q} f^{h} & \in H^{[q]}+H^{[q]} f^{h}+(H:(x, y))^{[q]} f^{2 h+1} \\
& \in H^{[q]}+H^{[q]} f^{h}+\left(H^{[q]}:\left(x^{q}, y^{q}\right)\right) f^{2 h+1}
\end{aligned}
$$

in $K\left[[x, y]\right.$. Since $f^{2 h+1} \in\left(x^{q}, y^{q}\right)$ (Lemma 1.10), we know that

$$
x u^{q} f^{h} \in H^{[q]}+H^{[q]} f^{h}+H^{[q]}=H^{[q]} .
$$

This implies that $x f^{h} \in H^{[q]}: u^{q}$. Since we are working over a regular ring, $x f^{h} \in$ $(H: u)^{[q]}$ (Corollary 1.14). Now $(x, y) \subseteq H: u$, and since $u \notin H, H: u=(x, y)$. So now we have that $x f^{h} \in(x, y)^{[q]}=\left(x^{q}, y^{q}\right)$. But $x f^{h} \notin\left(x^{q}, y^{q}\right)$. To see this expand $f^{h}=\left(x^{3}+y^{3}\right)^{h}$ using the binomial theorem. Thus $u z \notin I^{*}$.

Let $u \in\left(H:\left(x^{2}, x y, y^{2}\right)\right) \backslash(H:(x, y))$. Then $u z^{2} \in R_{2}$ represents an element of the socle mod $I$. We will use the grading and other arguments just as above. If $u z^{2} \in I^{*}$, then, using $x$ as a test element, and the fact that $f^{2 h} \in\left(x^{q}, y^{q}\right)$ (Lemma 1.10), we can show that $x u^{q} f^{2 h} \in H^{[q]}$. This implies that $x f^{2 h} \in$ $(H: u)^{[q]}$. Now $\left(x^{2}, x y, y^{2}\right) \subseteq H: u$, and since $u \notin H:(x, y), H: u \neq(x, y)$. Since $K[x, y] /\left(x^{2}, x y, y^{2}\right) \cong K+K x+K y$, we know that $H: u=\left(x^{2}, x y, y^{2}\right)$ or $\left(x, y^{2}\right)$ or $\left(x^{2}, y\right)$ or $\left(x^{2}, x y, y^{2}, x+\lambda y\right)$ where $\lambda \in K$. If we expand $f^{2 h}=\left(x^{3}+y^{3}\right)^{2 h}$, it is clear that $x f^{2 h} \notin\left(x^{2}, x y, y^{2}\right)^{[q]}$. Similarly, $x f^{2 h} \notin\left(x, y^{2}\right)^{[q]}$ and $x f^{2 h} \notin\left(x^{2}, y\right)^{[q]}$. Now suppose $x f^{2 h} \in\left(x^{2 q}, x^{q} y^{q}, y^{2 q}, x^{q}+\lambda^{q} y^{q}\right)$. Make a change of variables and replace $x$ by $x-\lambda y$. Now it is sufficient to show that

$$
(x-\lambda y)\left[(x-\lambda y)^{3}+y^{3}\right]^{2 h} \in\left((x-\lambda y)^{2 q},(x-\lambda y)^{q} y^{q}, y^{2 q}, x^{q}\right) .
$$

Expanding both sides shows that this cannot happen. Thus $u z^{2} \notin I^{*}$.

Proof of (3). Assume $q=3 h+1$. Let $u \in(H:(x, y)) \backslash H$. Then $u \in R_{0}$ represents an element of the socle mod $I$ (Lemma 1.7). We use the same method as in the proof of (2). If $u \in I^{*}$, then we use $x$ as a test element and multiply by $f^{h}$ to see that

$$
x u^{q} f^{h} \in H^{[q]} f^{h}+(H:(x, y))^{[q]} f^{2 h+1}+(H: f)^{[q]} f^{q} .
$$

Since $f^{2 h+1} \in\left(x^{q}, y^{q}\right)$ (Lemma 1.10), we can show that $x u^{q} f^{h} \in H^{[q]}$. This implies that $x f^{h} \in(H: u)^{[q]}$. As before $H: u=(x, y)$, and $x f^{h} \in\left(x^{q}, y^{q}\right)$, but $x f^{h} \notin$ $\left(x^{q}, y^{q}\right)$. Thus $u \notin I^{*}$.

Let $u \in\left(H:\left(x^{2}, x y, y^{2}\right)\right) \backslash(H:(x, y))$. Then $u z \in R_{1}$ represents an element of the socle mod $I$. If $u z \in I^{*}$, then we use $x$ as a test element and multiply by $f^{h}$. Since $f^{2 h} \in\left(x^{q}, y^{q}\right)$ (Lemma 1.10), we can show that $x u^{q} f^{2 h} \in H^{[q]}$. This implies that $x f^{2 h} \in(H: u)^{[q]}$. But this cannot happen by the second part of case (2). Thus $u z \notin I^{*}$.

Proof of (4). Let $q=3 h+1$. Let $u \in(H:(x, y)) \backslash H$. Then $u \in R_{0}$ represents an element of the socle mod $I$. If $u \in I^{*}$, then we use $x$ as a test element and multiply by $f^{h}$. Since $f^{2 h+1} \in\left(x^{q}, y^{q}\right)$ (Lemma 1.10), we can show that $x u^{q} f^{h} \in H^{[q]}$. This 
implies that $x f^{h} \in(H: u)^{[q]}$. As before, $H: u=(x, y)$, and $x f^{h} \in\left(x^{q}, y^{q}\right)$, but $x f^{h} \notin\left(x^{q}, y^{q}\right)$. Thus $u \notin I^{*}$.

Let $u \in\left(H:\left(x^{2}, x y, y^{2}\right)\right) \backslash(H:(x, y))$. Then $u z^{2} \in R_{2}$ represents an element of the socle $\bmod I$. If $u z^{2} \in I^{*}$, then we use $x$ as a test element and then multiply by $f^{h-2}$ to see that

$$
x u^{q} f^{3 h-2} \in H^{[q]} f^{h-2}+(H:(x, y))^{[q]} f^{2 h-2}+(H:(x, y))^{[q]} f^{3 h-2} .
$$

Since $f^{2 h-2} \in\left(x^{q}, y^{q}\right)$ (Lemma 1.12), we know that $x u^{q} f^{3 h-2} \in H^{[q]}$. This implies that $x f^{3 h-2} \in H^{[q]}: u^{q}$. As before we can show that $\left(x^{2}, x y, y^{2}\right) \subseteq H: u \subsetneq(x, y)$. As in the proof of (2), we know that $H: u=\left(x^{2}, x y, y^{2}\right)$ or $\left(x, y^{2}\right)$ or $\left(x^{2}, y\right)$ or $\left(x^{2}, x y, y^{2}, x+\lambda y\right)$ where $\lambda \in K$. Expand $f^{3 h-2}$ using the binomial theorem. We know that $\left(\begin{array}{c}3 h-2 \\ h-1\end{array}\right) \not \equiv 0 \bmod p$ by Proposition 1.12 , so $x f^{3 h-2} \notin\left(x^{2}, x y, y^{2}\right)^{[q]}$. Similarly, $x f^{3 h-2} \notin\left(x, y^{2}\right)^{[q]}$ and $x f^{3 h-2} \notin\left(x^{2}, y\right)^{[q]}$. Now suppose $x f^{3 h-2} \in$ $\left(x^{2 q}, x^{q} y^{q}, y^{2 q}, x^{q}+\lambda^{q} y^{q}\right)$. Make a change of variables and replace $x$ by $x-\lambda y$. An argument similar to the second part of the proof of (2) shows that this is impossible. Thus $u z^{2} \notin I^{*}$.

Proof of (5). Let $p=3 h+2$. Let $u \in(H:(x, y)) \backslash H$. Then $u z \in R_{1}$ represents an element of the socle $\bmod I$. If $u z \in I^{*}$, then, using $x$ as a test element, we must have $x u^{p} f^{h} \in H^{[p]}+H^{[p]} f^{h}+\left(H:\left(x^{2}, y\right)\right)^{[p]} f^{2 h+1}$ in $K[[x, y]]$ (Lemma 1.8). Let $A=K[[x, y]]$. Taking $p$ th roots of both sides yields

$$
x^{1 / p} u f^{h / p} \in H A^{1 / p}+H f^{h / p} A^{1 / p}+\left(H:\left(x^{2}, y\right)\right) f^{(2 h+1) / p} A^{1 / p} .
$$

We claim that $x^{1 / p} f^{h / p}$ is part of a free basis for $A^{1 / p}$ over $A$; equivalently $x f^{h}$ is part of a free basis for $A$ over $A^{p}=K\left[\left[x^{p}, y^{p}\right]\right]$. It is sufficient to see that $x f^{h}$ is not in the expansion of the maximal ideal of $A$ to $A^{p}$. If we expand $f^{h}=\left(x^{3}+y^{3}\right)^{h}$, it is clear that $x f^{h} \notin\left(x^{p}, y^{p}\right)$. Since $x^{1 / p} f^{h / p}$ is part of a free basis for $A^{1 / p}$ over $A$, we have an $A$-linear map $\theta: A^{1 / p} \rightarrow A$, sending $x^{1 / p} f^{h / p}$ to 1. It is clear that $\theta\left(f^{h / p} A^{1 / p}\right) \subseteq A$. If we expand $f^{(2 h+1) / p}$ and write it in terms of the basis, we see that $\theta\left(f^{(2 h+1) / p} A^{1 / p}\right) \subseteq\left(x^{2}, x y, y^{2}\right) A$. Thus applying $\theta$ to $(*)$ gives $u \in H+H+\left(H:\left(x^{2}, y\right)\right)\left(x^{2}, x y, y^{2}\right)$. Since $\left(x^{2}, x y, y^{2}\right) \subseteq\left(x^{2}, y\right)$, this implies that $u \in H$ which is a contradiction. Hence $u z \notin I^{*}$.

Now let $u \in\left(H:\left(x^{3}, x y, y^{2}\right)\right) \backslash\left(H:\left(x^{2}, y\right)\right)$. So $u z^{2} \in R_{2}$ represents an element of the socle $\bmod I$. Suppose $u z^{2} \in I^{*}$. The argument is the same as above except we use $y$ as a test element and show that there exists an $A$-linear map $\theta: A^{1 / p} \rightarrow A$, sending $y^{1 / p} f^{2 h / p}$ to 1 . This shows that $u z^{2} \notin I^{*}$.

In addition, in the following cases we can prove that if $u \in I^{*}$, then $u \in I^{F}$ for some but not all elements of the socle.

(2.2) Proposition. Let $I$ be a $\mathbb{Z}_{3}$-graded ideal of $K[[x, y, z]] /\left(x^{3}+y^{3}+z^{3}\right)$, where $K$ is a field of characteristic $p$ and $p \equiv 2 \bmod 3$.

(1) If $I=\left(H, H, H:\left(x^{2}, x y, y^{2}\right)\right)$, then $u z \notin I^{*}$ where $u \in H:(x, y)$.

(2) If $I=(H, J, J)$, then $u \in I^{*}$ implies $u \in I^{F}$ where $u \in(H:(x, y)) \backslash H$.

(3) If $I=(H, H, J)$, then $u z^{2} \in I^{*}$ implies $u z^{2} \in I^{F}$ where $u \in H:(x, y)$.

Proof. Proof of (1). Let $p=3 h+2$. Let $u \in(H:(x, y)) \backslash H$. Then $u z \in R_{1}$ represents an element of the socle $\bmod I$. Suppose $u z \in I^{*}$. We use the same argument as in 2.1 (5) with $x$ as a test element to show that $u z \notin I^{*}$. A similar 
technique does not work when trying to determine whether a socle element in $R_{2}$ is in $I^{*}$.

Proof of (2). Let $q=3 h+2$. Let $u \in((H:(x, y)) \cap J) \backslash H$, so $u \in R_{0}$ represents an element of the socle mod $I$. If $u \in I^{*}$, then, using $z$ as a test element, and the grading (Lemma 1.8), we determine that this is equivalent to having

$$
u^{q} \in H^{[q]}+J^{[q]} f^{h+1}+J^{[q]} f^{2 h+1}=H^{[q]}+J^{[q]} f^{h+1} .
$$

In order to have $u \in I^{F}$, we need $u^{q} \in I^{[q]}$ for $q \gg 0$, or equivalently,

$$
u^{q} \in H^{[q]}+J^{[q]} f^{h+1}+J^{[q]} f^{2 h+2}=H^{[q]}+J^{[q]} f^{h+1}
$$

(Lemma 1.8). As before, this technique provides no information about the contribution to the socle from $R_{2}$.

Proof of (3). Let $q=3 h+2$. Let $u \in\left((J:(x, y)) \cap\left(H:\left(x^{3}+y^{3}\right)\right)\right) \backslash H$, so $u z^{2} \in R_{2}$ represents the socle $\bmod I$. If $u z^{2} \in I^{*}$, then, using $z$ as a test element and the grading we see that this is equivalent to showing that

$$
u^{q} f^{2 h+1} \in H^{[q]}+H^{[q]} f^{h}+J^{[q]} f^{2 h+1}=H^{[q]}+J^{[q]} f^{2 h+1}
$$

in $K[[x, y]]$ (Lemma 1.8). In order to have $u z^{2} \in I^{F}$, we need

$$
u^{q} f^{2 h+1} \in H^{[q]}+H^{[q]} f^{h+1}+J^{[q]} f^{2 h+1}=H^{[q]}+J^{[q]} f^{2 h+1}
$$

in $K[[x, y]]$ (Lemma 1.8). So, if $u z^{2} \in I^{*}$, then $u z^{2} \in I^{F}$. As before, this technique provides no information about the contribution to the socle from $R_{1}$.

\section{Injective Modules over $R^{\infty}$}

We can study the question of whether $I^{*}=I^{F}$ in a ring $R$ by looking at injective modules over $R^{\infty}$. For example, if it were true that one could write the injective hull of $K$ over $R^{\infty}$ as a direct limit of cyclic modules, $R^{\infty} / I_{\nu}$, then we could reduce the problem for modules to studying the ideals $I_{\nu}$. At this point we can find a $\mathbb{Z}_{3}$-graded injective $R^{\infty}$-module that contains a copy of $K$. This is enough to give certain reductions in the problem of whether tight closure is the same as plus closure. We will use the following general lemma.

(3.1) Lemma. If $R$ is an A-algebra and $E$ is injective over $A$, then $\operatorname{Hom}_{A}(R, E)$ is an injective $R$-module.

Proof. See [E, Lemma A3.8].

(3.2) Comment. With $R=K[[x, y, z]] /\left(x^{3}+y^{3}+z^{3}\right), A=K$ and $E=K$, we see that $E_{R^{\infty}}=\operatorname{Hom}_{K}\left(R^{\infty}, K\right)$ is an injective $R^{\infty}$-module. In order to use this injective to reduce the problem of whether $I^{*}=I^{F}$ to the graded irreducible case, we will show that it contains a copy of $K$ and that it is $\mathbb{Z}_{3}$-graded.

(3.3) Lemma. Let $R=K[[x, y, z]] /\left(x^{3}+y^{3}+z^{3}\right)$ and $E_{R^{\infty}}=\operatorname{Hom}_{K}\left(R^{\infty}, K\right)$. Then $K \hookrightarrow E_{R^{\infty}}$.

Proof. Let $\phi \in \operatorname{Hom}_{K}\left(R^{\infty}, K\right)$ be the map $\phi: R^{\infty} \rightarrow R^{\infty} / m_{R^{\infty}} \hookrightarrow K$. Then $R^{\infty} \phi \cong K$, since $m_{R^{\infty}} \phi(x)=\phi\left(m_{R^{\infty}} x\right)=0$.

Next we would like to see that $E_{R^{\infty}}$ is $\mathbb{Z}_{3}$-graded.

(3.4) Lemma. Let $R=K[[x, y, z]] /\left(x^{3}+y^{3}+z^{3}\right)$ and $E_{R^{\infty}}=\operatorname{Hom}_{K}\left(R^{\infty}, K\right)$. Then $E_{R^{\infty}}$ is $\mathbb{Z}_{3}$-graded. 
Proof. Recall that the grading on $R$ extends to $R^{\infty}$ (see Section 1). Next, to see that $E_{R^{\infty}}$ is graded, write $R^{\infty}=R_{0}+R_{1}+R_{2}$. Then

$$
\operatorname{Hom}_{K}\left(R^{\infty}, K\right)=\operatorname{Hom}_{K}\left(R_{0}, K\right) \oplus \operatorname{Hom}_{K}\left(R_{1}, K\right) \oplus \operatorname{Hom}_{K}\left(R_{2}, K\right) .
$$

Let $E_{R^{\infty}}=W_{0}+W_{1}+W_{2}$ where $W_{i}=\operatorname{Hom}_{K}\left(R_{2 i}, K\right)$. Any subscripts that indicate a graded piece of a module or ring, e.g. $2 i$, will be reduced $\bmod 3$. If $\phi_{i} \in W_{i}$ and $r_{i} \in R_{i}$, then $\phi_{i}\left(r_{i}\right) \in K$ and $\phi_{i}\left(r_{j}\right)=0$ when $i \neq j$.

Let $f_{i} \in R_{i}$ and $\phi_{j} \in W_{j}$. We want to see that $f_{i} \phi_{j} \in W_{i+j}$. Recall that $W_{i+j}=$ $\operatorname{Hom}_{K}\left(R_{2(i+j)}, K\right)$, so we need to show that $f_{i} \phi_{j} \in \operatorname{Hom}_{K}\left(R_{2(i+j)}, K\right)$. Since $f_{i} \phi_{j}\left(r_{2(i+j)}\right)=\phi_{j}\left(f_{i} r_{2(i+j)}\right)$ and $f_{i} r_{2(i+j)} \in R_{i+2(i+j)}=R_{3 i+j}=R_{j}$, we know that $f_{i} \phi_{j}\left(r_{2(i+j)}\right) \in W_{i+j}$ as required. Similarly, if $k \neq 2(i+j)$, then $f_{i} \phi_{j}\left(r_{k}\right)=0$ and hence $f_{i} \phi_{j} \in W_{i+j}$.

(3.5) Theorem (Reduction to $\mathbb{Z}_{3}$-graded module case). Let

$$
R=K[[x, y, z]] /\left(x^{3}+y^{3}+z^{3}\right),
$$

where $K$ is a field of characteristic $p$. Let $I \subseteq R$ be an m-primary ideal such that $I^{*} \neq I^{F}$. Then there exist a $\mathbb{Z}_{3}$-graded $R$-module $M$ and an irreducible $\mathbb{Z}_{3}$-graded submodule $N$ such that $N^{*} \neq N^{F}$.

Proof. Suppose $I \subseteq R$ is an $m$-primary ideal such that $I^{*} \neq I^{F}$. Then there exists $u \in I^{*} R^{\infty} \backslash I R^{\infty}$. Expand $I R^{\infty}$ to an ideal of $R^{\infty}$ maximal with respect to not containing $u$. Then $u$ is the socle $\bmod I R^{\infty}$ and $I R^{\infty}$ is irreducible. To see that $u m_{R^{\infty}}=0$, note that $m_{R^{\infty}}=\bigcup m_{R^{1 / q}}$. Also, $u \in\left(I \cap R^{1 / q}\right)^{*}$ for some $q$. This implies that $m_{R^{1 / q}} u \subseteq I \cap R^{1 / q}$. Thus $m_{R^{\infty}} u \subseteq I R^{\infty}$.

Let $E_{R^{\infty}}$ be a $\mathbb{Z}_{3}$-graded injective $R^{\infty}$-module that contains a copy of $K$. We know one exists by Lemmas 3.3 and 3.4. We have an injective map $R^{\infty} / I R^{\infty} \rightarrow$ $E_{R^{\infty}}$ sending 1 to $\alpha$. We can find a finitely generated ideal $I_{0} \subseteq R^{1 / q}$ such that $u \in I_{0}^{* f g}$, the finitistic tight closure. Here $I_{0}^{* f g}=\bigcup_{J}\left(I_{0} \cap J\right)^{*}$ where $J$ ranges over all finitely generated ideals of $R^{\infty} / I R^{\infty}$. Let $\tilde{u}$ be the image of $u$ in $R^{1 / q} / I_{0}$. Let $M$ be the submodule of $E_{R^{\infty}}$ generated by $\alpha$. Then we have a map $R^{1 / q} / I_{0} \longrightarrow M$. $M$ is a finitely generated $R^{1 / q}$-module that contains the image of $R^{1 / q} / I_{0}$ and is graded. It is still true that $\tilde{u} \in I_{0}^{*}$ in $M$ since $u \in 0^{*}$ in $E_{R^{\infty}}$. If $u \in 0^{F}$ in $M$, then we would have $u \in 0^{F}$ in $E_{R^{\infty}}$ and an element is in $0^{F}$ in an $R^{\infty}$-module if and only if it is zero. Thus $u \notin 0^{F}$ in $M$.

\section{IRREDUCiBle IDEALS}

As we saw in Section 3, we can reduce the question of whether $I^{*}=I^{F}$ in $R=K[[x, y, z]] /\left(x^{3}+y^{3}+z^{3}\right)$ to the graded irreducible module case. Given this reduction, it seems likely that understanding the graded irreducible ideal case will be helpful. In this section we will show that $I^{*}=I^{F}$ for most $\mathbb{Z}_{3}$-graded irreducible ideals in $R$ when $K$ has characteristic $p$ and $p \equiv 2 \bmod 3$. In the course of proving the main result, Theorem 4.5, we develop a number of techniques for determining when an element of the socle is in the tight closure or the Frobenius closure of a given ideal.

Preliminary Techniques. The following proposition provides a useful tool for determining whether or not a given irreducible $m$-primary ideal, $I$, is tightly closed. If we can find an irreducible ideal contained in $I$ which is tightly closed, then we 
know that $I$ is also tightly closed. Similarly, if we can find an irreducible ideal containing $I$ which is not tightly closed, then we know that $I$ is not tightly closed.

(4.1) Proposition. Let $R$ be a local Gorenstein ring. Let $m$ be the maximal ideal of $R$ and let $J$ and $I$ be irreducible m-primary ideals of $R$ with $J \subseteq I$. Then $R / I \hookrightarrow R / J$, and if $I^{*} \neq I$, then $J^{*} \neq J$. Also, if $I^{F} \neq I$, then $J^{F} \neq J$.

Proof. Since $I$ and $J$ are $m$-primary, $R / I$ and $R / J$ are zero-dimensional. As $I$ and $J$ are irreducible and $m$-primary, $\operatorname{dim}_{K} \operatorname{Soc} R / J=1$ and $R / J$ is Gorenstein, and similarly for $R / I$. So $R / J$ is a zero-dimensional Gorenstein local ring, which implies that $R / J$ is injective as a module over itself and $R / J \cong E_{R / J}(K)$. Similarly, $R / I \cong E_{R / I}(K)$. So $\operatorname{Ann}_{R / J} I \cong \operatorname{Ann}_{E_{R / J}(K)} I \cong E_{(R / J) / I}(K) \cong E_{R / I}(K) \cong$ $R / I$, and thus $\operatorname{Ann}_{(R / J)} I \cong R / I$. Composing this isomorphism with the natural inclusion $\operatorname{Ann}_{(R / J)} I \hookrightarrow R / J$ gives the inclusion $\phi: R / I \rightarrow R / J$. We also know that $\phi\left((0)_{R / I}^{*}\right) \subseteq(0)_{R / J}^{*}$. If $I^{*} \neq I$, then $(0)_{R / I}^{*}=I^{*} / I \neq 0$, and so $(0)_{R / J}^{*} \neq 0$. Then $J^{*} / J \neq 0$ and $J^{*} \neq J$ as required. The same argument applies for $I^{F}$ and $J^{F}$ since $\phi\left((0)_{R / I}^{F}\right) \subseteq(0)_{R / J}^{F}$.

In fact, even if one or both of $I$ and $J$ is not irreducible, if we can show that we have an injection $R / I \hookrightarrow R / J$, then $J^{*}=J$ implies that $I^{*}=I$. The following lemma gives a criterion for when such an injection exists.

(4.2) Lemma. Let $R$ be a Noetherian ring. Let $I$ and $J$ be ideals of $R$ with $J \subseteq I$, $I$ irreducible and let $u$ be the socle mod $I$. Then $R / I \hookrightarrow(R / J)^{h}$ if and only if there exists $v \in R$ such that $v I \subseteq J$ and $v u \notin J$. If, in addition, $J=J^{*}$, then $I=I^{*}$.

Proof. Let $u_{1}, \ldots, u_{h}$ generate $J: I$. Let $\bar{u}_{1}, \ldots, \bar{u}_{h}$ be the images of the generators in $R / J$. Then $\bar{u}_{1}, \ldots, \bar{u}_{h}$ generate $(J: I) / J \cong \operatorname{Ann}_{R / J} I$. We have a map $R \rightarrow$ $(R / J)^{h}$ taking $\bar{r}$ to $\left(r \bar{u}_{1}, \ldots, r \bar{u}_{h}\right)$. Now $\bar{r}$ gets mapped to 0 if and only if $r(J: I) \subseteq$ $J$. This is equivalent to having $r \in J:(J: I)$. So the map is injective if and only if $I=J:(J: I)$. This is equivalent to having $u \notin J:(J: I)$ or $u(J: I) \nsubseteq J$. Finally, this is true if and only if there exists $v \in J: I$ such that $u v \notin J$.

Suppose $u \in 0_{R / I}^{*}$. Then the image of $u$ is contained in $0_{R / J}^{*}$. Thus if $J$ is tightly closed, so is $I$.

(4.3) Proposition. Let $R=K[[x, y, z]] /\left(x^{3}+y^{3}+z^{3}\right)$, where $K$ is a field of characteristic $p$ and $p \equiv 2 \bmod 3$. Let $I$ be an irreducible $m$-primary ideal of $R$ and let $u$ represent the socle mod $I$. If $I \subseteq(x, y)$, then $u \in I^{F}$. Let $(f, g)$ be generated by a system of parameters. If $I \subseteq(f, g)$, then $u \in I^{F}$.

Proof. Since $I$ and $(x, y)$ are both irreducible $m$-primary ideals, we have an injection $R /(x, y) \hookrightarrow R / I$ sending $z^{2}$, the socle in $R /(x, y)$, to $u$ (Proposition 4.1). It is enough to see that $z^{2} \in(x, y)^{F}$, for then $u \in I^{F}$. For this it is sufficient to show that $z^{2 p}$ is contained in $\left(x^{p}, y^{p}\right)$. Let $p=3 h+2$. Using the basic relation in $R$ and the $\mathbb{Z}_{3}$-grading it is sufficient to show that $\left(x^{3}+y^{3}\right)^{2 h+1} \in\left(x^{p}, y^{p}\right)$ (Lemma 1.8). This is routine if we expand using the binomial theorem. Thus $z^{2} \in(x, y)^{F}$.

Let $v$ represent the socle in $R /(f, g)$. Since $I$ and $(f, g)$ are both irreducible $m$ primary ideals, we have an injection $R /(f, g) \hookrightarrow R / I$ sending $v$ to $u$ (Proposition 4.1). It is enough to see that $v \in(x, y)^{F}$, for then $u \in I^{F}$. We know that $\left(f^{q}, g^{q}\right) \subseteq$ $(x, y)$ for some $q$. The socle $\bmod \left(f^{q}, g^{q}\right)$ is $f^{q-1} g^{q-1} v$. Since $\left(f^{q}, g^{q}\right)$ is an $m$ primary irreducible ideal contained in $(x, y)$, we know that $f^{q-1} g^{q-1} v \in\left(f^{q}, g^{q}\right)^{F}$. 
This implies that $f^{(q-1) Q} g^{(q-1) Q} v^{Q} \in\left(f^{q Q}, g^{q Q}\right)$ for some $Q=p^{e}$. Dividing by powers of $f$ and $g$ yields $v^{Q} \in\left(f^{Q}, g^{Q}\right)$, and hence $v \in(f, g)^{F}$.

Classification of Irreducibles. The $\mathbb{Z}_{3}$-grading on $R$ allows us to characterize the irreducible ideals.

(4.4) Proposition. Let $I$ be an irreducible m-primary $\mathbb{Z}_{3}$-graded ideal of $K[[x, y, z]] /\left(x^{3}+y^{3}+z^{3}\right)$, where $K$ is a field of characteristic $p$. Then I corresponds to one of the following triples of ideals in $K[[x, y]]$ where $H$ is an irreducible m-primary ideal of $K[[x, y]]$ and $f=x^{3}+y^{3}:(H, H, H),(H, H: f, H: f)$, $(H, H, H: f)$.

Proof. We know that $\left(H_{0}+H_{1} z+H_{2} z^{2}\right):(x, y, z)$ can be decomposed into graded pieces as follows:

$$
\left(\left(H_{0}:(x, y)\right) \cap H_{2}\right)+\left(\left(H_{1}:(x, y)\right) \cap H_{2}\right) z+\left(\left(H_{2}:(x, y)\right) \cap\left(H_{0}:\left(x^{3}+y^{3}\right)\right)\right) z^{2}
$$

(Lemma 1.7). Suppose $u$, the socle $\bmod I$, is contained in $R_{0}$, the zero graded piece of $R$. Then in order for $I$ to have a one-dimensional socle, there must be no contribution from $R_{1}$ or $R_{2}$. This requires that $\left(H_{1}:(x, y)\right) \cap H_{2}=H_{1}$ and $\left(H_{2}:(x, y)\right) \cap\left(H_{0}: f\right)=H_{2}$. These conditions imply that $H_{1}=H_{2}$ and $H_{2}=$ $H_{0}: f$, respectively. To see this, just note that if $H_{1}$ were strictly contained in $H_{2}$, since $H_{1}:(x, y)$ is strictly larger than $H_{1}$, their intersection would strictly contain $H_{1}$. In other words, $I$ corresponds to the triple $\left(H_{0}, H_{0}: f, H_{0}: f\right)$. The annihilator of $(x, y, z)$ is now $\left(H_{0}:(x, y)\right) \cap\left(H_{0}: f\right)$. Since $(f) \subseteq(x, y)$, we know that $\left(H_{0}:(x, y)\right) \subseteq\left(H_{0}: f\right)$, and so the intersection is just $H_{0}:(x, y)$. The socle is then $\left(H_{0}:(x, y)\right) \backslash H_{0}$ or just the socle $\bmod H_{0}$ in $K[[x, y]]$. Thus, if $H_{0}$ is an irreducible ideal of $K[[x, y]]$, then $I$ has a one-dimensional socle and is irreducible. Similar arguments are used if the socle mod $I$ is contained in $R_{1}$ or $R_{2}$.

Tight Closure and Frobenius Closure of Irreducible Ideals. Now we can prove the main result of this section.

(4.5) Theorem. Let $I$ be an irreducible m-primary $\mathbb{Z}_{3}$-graded ideal of $K[[x, y, z]] /\left(x^{3}+y^{3}+z^{3}\right)$, where $K$ is a field of characteristic $p$ and $p \equiv 2 \bmod 3$. Let $f=\left(x^{3}+y^{3}\right)$. If I has any of the following forms, then $I^{*}=I^{F}$.

(1) $(H, H, H)$,

(2) $(H, H: f, H: f)$,

(3) $(H, H, H: f)$ and $f \notin H$,

(4) $(H, H, H: f)$ and $f \in H$ and $H$ contains an element with a linear form.

Proof of (1)-(3). First observe that $(H, H, H) \subseteq(x, y)$. The ideals $(H, H: f, H: f)$ and $(H, H, H: f)$ are also contained in $(x, y)$ so long as $f \notin H$. If $f \in H$, then $H: f=K[[x, y]]=A$. In that case, $(H, H: f, H: f)=(H, A, A)=H+A z$ and $(H, H, H: f)=(H, H, A)=H+A z^{2}$. When the ideals are contained in $(x, y)$ we know that $I^{*}=I^{F}$ by Proposition 4.3. In fact, we know that $I^{*} \neq I$ in those cases.

We will now consider the case $I=(H, H: f, H: f)$ where $f \in H$. As noted before, $I=H+A z$ in this case. Let $q=3 h+1$. Suppose $u \in I^{*}$. Then, using $z$ as a test element, and the grading (Lemma 1.9), we see that this is equivalent to having $u^{q} \in H^{[q]}+\left(f^{h+1}\right)+\left(f^{2 h+1}\right)$ in $K[[x, y]]$ which implies that $u^{q} \in H^{[q]}+\left(f^{h+1}\right)$. This, however, is exactly what is needed to have $u^{q} \in I^{[q]}$ (Lemma 1.9). Thus $u \in I^{F}$. 
The proof of (4) requires several different techniques. We begin with an analysis of the possible forms for $H$.

(4.6) Lemma. Let $R=K[[x, y, z]] /\left(x^{3}+y^{3}+z^{3}\right)$, where $K$ is a field of characteristic $p$ and $p \equiv 2 \bmod 3$. Let $I$ be a $\mathbb{Z}_{3}$-graded irreducible ideal of the form $(H, H, H: f)$ with $f=\left(x^{3}+y^{3}\right) \in H$. If $H$ contains an element with a linear form, then $H$ has one of the following forms:

(1) $(x, y)$,

(2) $\left(x^{2}, y-c x\right), c \in K \backslash\{0\}$,

(3) $\left(x^{k}, y+x\right), k \geq 3$,

(4) $\left(x^{k}, y+x-d x^{k-1}\right), k \geq 3, d \in K \backslash\{0\}$.

Proof. Let $q=3 h+1$. We can assume that $H \nsubseteq\left(x, y^{3}\right)$ in $K[[x, y]]$; otherwise $I$ would be contained in a parameter ideal of $R$ and we would done by Proposition 4.3. Suppose an element of $H$ has a term $\alpha y+\cdots$ with $\alpha \neq 0$. Using Weierstrass preparation, we can find a unique monic associate $u=y-g(x)$. Now $K[[x, y]] / u \cong$ $K[[x]]$, a principal ideal domain. $H /(u)$ is an ideal of $K[[x]]$, and since $K[[x]]$ is a PID, $H /(u)=x^{k}$ for some $k$. Lifting back to $K[[x, y]]$ we see that $H=\left(x^{k}, y-g(x)\right)$. We can also assume that $x^{k} \notin(y-g(x), z)$; otherwise $I$ would be contained in the ideal $(y-g(x), z)$ which is a parameter ideal. Suppose $x^{k} \notin(y-g(x), z)$ in $R$. Using the $\mathbb{Z}_{3}$-grading (Lemma 1.9) we see that this is equivalent to having $x^{k} \notin\left(y-g(x), x^{3}+y^{3}\right)$ in $K[[x, y]]$, which is equivalent to having $x^{k} \notin\left(x^{3}+g(x)^{3}\right)$ in $K[[x, y]]$ modulo $u=y-g(x)$. In order to have $x^{k} \notin\left(x^{3}, g(x)^{3}\right)$, we need the order of $x^{3}+g(x)^{3}$ to be greater than $k$. Assume $\operatorname{ord}_{x} g(x) \geq 2$ or $c \neq-1$ where $g(x)=c x+\cdots$. If $k=1$, then $H=(x, y-g(x))=(x, y)$. If $k=2$, then $H=\left(x^{2}, y-g(x)\right)=\left(x^{2}, y-c x\right)$.

Now suppose that $k>2$. We still need the order of $x^{3}+g(x)^{3}$ to be greater than $k$. We can assume that $\operatorname{ord}_{x} g(x)=1$ and $g(x)=-x+d x^{h}+\cdots$. Then $x^{3}+g(x)^{3}=3 d x^{2+h}+$ lower degree terms. So we need $h+2>k$. If $k<h+1$, then $\left(x^{k}, y-g(x)\right)=\left(x^{k}, y+x\right)$. If $k=h+1$, then $\left(x^{k}, y-g(x)\right)=\left(x^{k}, y+x-d x^{k-1}\right)$. In each case $k \geq 3$.

We can now deal with these cases separately.

(4.7) Remark. Let $R$ be a Noetherian ring and $m$ a maximal ideal. If $I$ is an $m$ primary ideal of $R$, then $R / I \cong \hat{R} / I \hat{R}$. If we are interested in whether $u \in I \hat{R}$, it is sufficient to check whether $u \in I$. We will make use of this idea in several of the following propositions by reducing questions about ideal membership in $K[[x, y]]$ to the polynomial ring $K[x, y]$.

(4.8) Proposition. Let $R=K[[x, y, z]] /\left(x^{3}+y^{3}+z^{3}\right)$, where $K$ is a field of characteristic $p$ and $p \equiv 2 \bmod 3$.

(1) Let $I=\left(x, y, z^{2}\right)$. Then $I^{*}=I^{F}=I$.

(2) Let $I=\left(x^{2}, y-c x, z^{2}\right), c \in K \backslash\{0\}$. Then $I^{*}=I^{F}=I$.

(3) Let $I=\left(x^{k}, y+x, z^{2}\right)$ with $k \geq 3$. Then $I^{*}=I^{F}=I$.

Proof. Let $p=3 h+2$ and $f=x^{3}+y^{3}$.

(1) The socle mod $I$ is $z$. Using $z$ as a test element, it suffices to see that $z z^{p} \notin$ $\left(x^{p}, y^{p}, z^{2 p}\right)$. Suppose $z z^{p} \in\left(x^{p}, y^{p}, z^{2 p}\right)$. Using the basic relation in $R$, and the $\mathbb{Z}_{3^{-}}$ grading (Lemma 1.8), we see that this is equivalent to having $f^{h+1} \in\left(x^{p}, y^{p}, f^{2 h+2}\right)$ in $K[[x, y]]$. A degree argument shows that this cannot hold. 
(2) The socle $\bmod I$ is $x z$. Using $z$ as a test element, it suffices to see that $z(x z)^{p} \notin\left(x^{2 p}, y^{p}-c^{p} x^{p}, z^{2 p}\right)$. Suppose $z(x z)^{p} \in\left(x^{2 p}, y^{p}-c^{p} x^{p}, z^{2 p}\right)$. Using the basic relation in $R$ and the $\mathbb{Z}_{3}$-grading (Lemma 1.8) we see that this is equivalent to having $x^{p} f^{h+1} \in\left(x^{2 p}, y^{p}-c^{p} x^{p}, f^{2 h+2}\right)$ in $K[[x, y]]$. The degree of $x^{p} f^{h+1}$ is $2 p+1$, while the degree of $f^{2 h+2}$ is $2 p+2$. Since we are in the homogeneous case, we may conclude that $x^{p} f^{h+1}=\left(a_{1} x+a_{2} y\right) x^{2 p}+B\left(y^{p}-c^{p} x^{p}\right)$ where $a_{1}, a_{2} \in K$ and $B \in K[x, y]$ (4.7). Since $x^{p} f^{h+1}$ has no term with the degree of $x$ less than $p, B=\left(b_{1} x^{p+1}+b_{2} x^{p} y\right), b_{1}, b_{2} \in K$. Expanding $x^{p} f^{h+1}$ shows that the equality cannot hold.

(3) The socle mod $I$ is $x^{k-1} z$. Using $z$ as a test element, it suffices to see that $z\left(x^{k-1} z\right)^{p} \notin\left(x^{k p}, y^{p}+x^{p}, z^{2 p}\right)$. Suppose $z\left(x^{k-1} z\right)^{p} \in\left(x^{k p}, y^{p}+x^{p}, z^{2 p}\right)$. Using the basic relation in $R$ and the $\mathbb{Z}_{3}$-grading (Lemma 1.8) shows that this is equivalent to having $x^{(k-1) p} f^{h+1} \in\left(x^{k p}, y^{p}+x^{p}, f^{2 h+2}\right)$ in $K[[x, y]]$. Since we are in the homogeneous case,

$$
x^{(k-1) p} f^{h+1}=\left(a_{1} x+a_{2} y\right) x^{k p}+B\left(x^{p}+y^{p}\right)+C f^{2 h+2}
$$

where $a_{1}, a_{2} \in K$ and $B, C \in K[x, y]$ (4.7). Let $x^{3}+y^{3}=(x+y) Q$, where Q is the quadratic form $x^{2}-x y+y^{2}$. It is clear that $a_{1}=a_{2}$ since $(x+y)$ must divide the term $\left(a_{1} x+a_{2} y\right) x^{k p}$. So

$$
x^{(k-1) p}(x+y)^{h+1} Q^{h+1}=a(x+y) x^{k p}+B(x+y)^{p}+C(x+y)^{2 h+2} Q^{2 h+1} .
$$

Dividing both sides by $(x+y)$ implies that $(x+y)^{h}$ divides $a x^{k p}$ which is clearly false.

(4.9) Proposition. Let $R=K[[x, y, z]] /\left(x^{3}+y^{3}+z^{3}\right)$, where $K$ is a field of characteristic $p$ and $p \equiv 2 \bmod 3$. Let $I=\left(x^{k}, x+y-d x^{k-1}, z^{2}\right), k \geq 3, d \in K \backslash\{0\}$. Then $I^{*}=I^{F}=I$.

Proof. The socle mod $I$ is $x^{k-1} z$. Using $z$ as a test element, it suffices to show that $z x^{(k-1) p} z^{p} \notin\left(x^{k p}, x^{p}+y^{p}-d^{p} x^{(k-1) p}, z^{2 p}\right)$. We will reduce to the case $d=1$. Apply the following map to $R: x \rightarrow \lambda x, y \rightarrow \lambda y$, and $z \rightarrow \lambda z$, where $\lambda \in K$. Then

$$
z x^{k-1} z \in\left(x^{k}, x+y-d x^{k-1}, z^{2}\right)^{*}
$$

if and only if

$$
\lambda^{k} z x^{k-1} \in\left(\lambda^{k} x^{k}, \lambda x+\lambda y-\lambda^{k-1} d x^{k-1}, \lambda^{2} z^{2}\right)^{*} .
$$

By factoring out the $\lambda \mathrm{s}$, we are left with $z x^{k-1} \in\left(x^{k}, x+y-\lambda^{k-2} d x^{k-1}, z^{2}\right)^{*}$. If $d \neq 0$, let $\lambda=d^{-1 /(k-2)}$. So if $x^{k-1} z$ is in the tight closure of the ideal for one value of $d \neq 0$, then it is in for all $d \neq 0$. We have reduced to the case where $I=\left(x^{k}, x+y-x^{k-1}, z^{2}\right)$. By Lemma 4.2 it is enough to find an ideal $J \subseteq I$ such that $J$ is tightly closed and $R / I \hookrightarrow R / J$. Let

$$
J_{0}=\left((x+y)^{2}, x^{2 k-2},(x+y) x^{k}, x^{2 k-1},(x+y) z^{2}, x^{k-1} z^{2}\right) .
$$

The desired $J$ is $J_{0}{ }^{*}$. In order to show that $R / I \hookrightarrow R / J_{0}^{*}$, it is sufficient to find $v \in J_{0}^{*}: I$ such that $v u \notin J_{0}^{*}$ where $v$ is the socle $\bmod I$ (Lemma 4.2).

First we want to see that $J_{0}{ }^{*} \subseteq I$. Let $J_{1}=\left(y(x+y), x(x+y), x^{k}, z^{2}\right)$. The socle mod $J_{1}$ is generated by $(x+y) z$ and $x^{k-1} z$. We would like to show that $J_{1}=J_{1}{ }^{*}$. We know that $(x+y) z \notin J_{1}{ }^{*}$ by a degree argument [Sm3, Theorem 2.2]. To show that $x^{k-1} z \notin J_{1}{ }^{*}$ we will consider the ideal $J_{2}=\left(x+y, x^{k}, z^{2}\right)$. We know that $x^{k-1} z \notin J_{2}{ }^{*}$ and $J_{2}=J_{2}{ }^{*}$ by a previous case (Proposition 4.8 (3)). As $J_{1} \subseteq J_{2}$ and $x^{k-1} z \notin J_{2}{ }^{*}$, we may conclude that $x^{k-1} z \notin J_{1}{ }^{*}$. Thus $J_{1}=J_{1}{ }^{*}$. We 
also know that $J_{0} \subseteq J_{1}$ implies $J_{0}{ }^{*} \subseteq J_{1}{ }^{*}$ [HH1, Proposition 4.1]. Now we have $J_{0}{ }^{*} \subseteq J_{1}{ }^{*}=J_{1} \subseteq I$, which guarantees that $J_{0}{ }^{*} \subseteq I$.

Next we would like to show that $x+y+x^{k-1} \in J_{0}{ }^{*}: I$. First we note that

$$
\left(x+y+x^{k-1}\right) I \subseteq\left((x+y) x^{k}, x^{2 k-1},(x+y)^{2}-x^{2 k-2},(x+y) z^{2}, x^{k-1} z^{2}\right) .
$$

Certainly $\left((x+y) x^{k}, x^{2 k-1},(x+y)^{2}-x^{2 k-2},(x+y) z^{2}, x^{k-1} z^{2}\right) \subseteq J_{0} \subseteq J_{0}^{*}$.

Recall that $x^{k-1} z$ is the socle mod $I$. We want to show that $\left(x+y+x^{k-1}\right) x^{k-1} z \notin$ $J_{0}{ }^{*}$. Since $J_{0}$ and hence $J_{0}^{*}$ are homogeneous, it is enough to show that $(x+$ $y) x^{k-1} z \notin J_{0}{ }^{*}$. Using $z$ as a test element, it suffices to see that $z(x+y)^{p} x^{(k-1) p} z^{p} \notin$ $J_{0}{ }^{[p]}$. Suppose $z(x+y)^{p} x^{(k-1) p} z^{p} \in J_{0}{ }^{[p]}$. Using the basic relation in $R$ and the $\mathbb{Z}_{3}$-grading (Lemma 1.8) shows that this is equivalent to having

$(x+y)^{p} x^{(k-1) p} f^{h+1} \in\left((x+y)^{p} x^{k p}, x^{(2 k-2) p},(x+y)^{2 p},(x+y)^{p} f^{h+2}, x^{(k-1) p} f^{h+2}\right)$.

Since we are in the homogeneous case, routine degree arguments show that

$$
(x+y)^{p} x^{(k-1) p} f^{h+1} \in\left((x+y)^{p} x^{k p},(x+y)^{2 p},(x+y)^{p} f^{h+2}\right)
$$

as long as $k>3$. Dividing by $(x+y)^{p}$ yields $x^{(k-1) p} f^{h+1} \in\left(x^{k p},(x+y)^{p}, f^{h+2}\right)$. But this is equivalent to having $x^{k-1} z \in\left(x^{k},(x+y), z^{2}\right)^{*}$. We know that $x^{k-1} z \notin$ $\left(x^{k}, x+y, z^{2}\right)^{*}$ by a previous result (Proposition $\left.4.8(3)\right)$.

Let $k=3$ and suppose that

$$
(x+y)^{p} x^{2 p} f^{h+1} \in\left((x+y)^{p} x^{3 p}, x^{4 p},(x+y)^{2 p},(x+y)^{p} f^{h+2}, x^{2 p} f^{h+2}\right) .
$$

The degree of $(x+y)^{p} x^{2 p} f^{h+1}$ is $4 p+1$. Since we are in the homogeneous case, this implies that

$$
(x+y)^{p} x^{2 p} f^{h+1}=A(x+y)^{p}+\left(\beta_{1} x+\beta_{2} y\right) x^{4 p}+C x^{2 p} f^{h+2}
$$

where $\beta_{1}, \beta_{2} \in K$ and $A, C \in K[x, y]$ (4.7). But this implies that $(x+y)^{h+2}$ divides $\left(\beta_{1} x+\beta_{2} y\right) x^{4 p}$ which is impossible.

So with $v=x+y+x^{k-1}$, we have $v \in J_{0}^{*}: I$ and $x^{k-1} z v \notin J_{0}^{*}$. This is enough to show $R / I \hookrightarrow R / J_{0}^{*}$ by Lemma 4.2 . Since $J_{0}^{*}$ is tightly closed, we know that $I$ is tightly closed, also by Lemma 4.2 .

In addition to the cases where $I \subseteq(x, y)$, we can determine whether or not an irreducible ideal is tightly closed, not just that $I^{*}=I^{F}$, in the following cases.

(4.10) Proposition. Let $R=K[[x, y, z]] /\left(x^{3}+y^{3}+z^{3}\right)$, where $K$ is a field of characteristic $p$. Let $I$ be an irreducible $\mathbb{Z}_{3}$-graded ideal of the form $(H, H: f, H: f)$, where $f=x^{3}+y^{3} \in H$, and $H$ is generated by elements whose leading forms are relatively prime quadratic forms. Then $I=I^{*}$.

Proof. $I$ is of the form $\left(Q_{1}+C_{1}, Q_{2}+C_{2}, z\right)$. Here we mean the ideal generated by $Q_{1}+C_{1}, Q_{2}+C_{2}$, and $z$, not a triple of ideals. Let $Q_{3}$ be the third independent quadratic form. By considering the associated graded ring we can see that $K[[x, y]] /\left(Q_{1}+C_{1}, Q_{2}+C_{2}\right)$ has dimension four over $K$, and it follows that $1, x, y, Q_{3}$ give a basis. Everything of degree three or more will be in $H$ and $Q_{3}$ will represent the socle $\bmod I$. This also guarantees that $f \in H$. We would like to show that $Q_{3} \notin\left(Q_{1}+C_{1}, Q_{2}+C_{2}, z\right)^{*}$. Using the grading and $x$ as a test element, it is sufficient to show that $x Q_{3}^{p} \notin\left(Q_{1}^{p}, Q_{2}^{p}, f^{h+1}\right)$. This is equivalent to showing that $x Q_{3}^{p}+L_{1} Q_{1}^{p}+L_{2} Q_{2}^{p}$ is not divisible by $f^{h+1}$ where $L_{1}$ and $L_{2}$ are linear forms. We will dehomogenize the equation by setting $y=1$. If $x Q_{3}^{p}+L_{1} Q_{1}^{p}+L_{2} Q_{2}^{p}$ is divisible by $f^{h+1}$, then $x{\overline{Q_{3}}}^{p}+{\overline{L_{1} Q_{1}}}^{p}+{\overline{L_{2} Q_{2}}}^{p}$ is divisible by $\bar{f}^{h+1}$. This implies 
the derivative with respect to $x$ is divisible by $\bar{f}^{h}$. Using the fact that we are in characteristic $p$, we see that the derivative is ${\overline{Q_{3}}}^{p}+{\overline{L_{1}}}^{\prime}{\overline{Q_{1}}}^{p}+{\overline{L_{2}}}^{\prime}{\overline{Q_{2}}}^{p}$. So we need that $\left(\overline{Q_{3}}+\left(\overline{L_{1}}\right)^{1 / p} \overline{Q_{1}}+\left(\overline{L_{2}}\right)^{1 / p} \overline{Q_{2}}\right)^{p}$ is divisible by $\bar{f}^{h}$. If we rewrite $\bar{f}^{h}$ as $(x-1)^{h}(x-\omega)^{h}(x-\bar{\omega})^{h}$, we conclude that all three linear factors of $\bar{f}$ divide $\left(\overline{Q_{3}}+\left(\overline{L_{1}}\right)^{1 / p} \overline{Q_{1}}+\left(\overline{L_{2}}\right)^{1 / p} \overline{Q_{2}}\right)$. Since $\overline{Q_{1}}$ and $\overline{Q_{2}}$ are still independent over $K$, this cannot happen.

(4.11) Comment. Let $(H, z)$ and $\left(H, z^{2}\right)$ be two irreducible $m$-primary ideals of $K[[x, y, z]] /\left(x^{3}+y^{3}+z^{3}\right)$. Since $\left(H, z^{2}\right) \subseteq(H, z)$, we know that if $\left(H, z^{2}\right)$ is tightly closed, then so is $(H, z)$ (Proposition 4.1). In particular, if $I=\left(x, y, z^{2}\right)$, $\left(x^{2}, y-c x, z^{2}\right),\left(x^{k}, y+x, z^{2}\right)$, or $\left(x^{k}, x+y-x^{k-1}, z^{2}\right)$, we know that $I=I^{*}$. So if $I=(x, y, z),\left(x^{2}, y-c x, z\right),\left(x^{k}, y+x, z\right)$, or $\left(x^{k}, x+y-x^{k-1}, z\right)$, we know that $I=I^{*}$ also.

Next we classify the cases of $m$-primary irreducible $\mathbb{Z}_{3}$-graded ideals not included in Theorem 4.5. To do this we need the following proposition which gives a characterization of the $m$-primary irreducible ideals in $K[[x, y]]$.

(4.12) Lemma. Let $A=K[[x, y]]$. Let $I$ be an irreducible m-primary ideal in $A$. Then $I$ is generated by parameters.

Proof. First note that $I$ is a height two ideal and the quotient, $A / I$, is CohenMacaulay and has finite projective dimension. This means that $A / I$ must have a resolution that looks like $0 \rightarrow A^{r-1} \rightarrow A^{r} \rightarrow A \rightarrow A / I \rightarrow 0$ where the entries of the matrix of the map from $A^{r}$ to $A$ can be taken to be minimal generators of $I$. Then $I$ must be the ideal generated by the $r-1$ size minors of of the second matrix. This implies that the type of $A / I$ is one smaller than the number of generators of $I$. Since $A / I$ has type one, we must have $r=2$.

We are now able to classify the remaining cases.

(4.13) Proposition. Let $R=K[[x, y, z]] /\left(x^{3}+y^{3}+z^{3}\right)$ and $A=K[[x, y]]$, where $K$ is a field of characteristic $p \neq 3$. Let $I$ be an m-primary irreducible $\mathbb{Z}_{3}$-graded ideal of $R$ corresponding to the triple of ideals $(H, H, H: f)$, where $f=x^{3}+y^{3} \in H$. Suppose $H$ does not contain an element with a linear leading form. Then I has one of the following forms:

(1) $I=\left(Q_{1}, Q_{2}, z^{2}\right)$ where $Q_{1}, Q_{2}$ are relatively prime quadratic forms in $A$;

(2) $I=\left(L_{1}^{2}+C, L_{1} L_{2}+D, z^{2}\right)$ where $L_{1}$ and $L_{2}$ are independent linear forms, $L_{1}$ divides $f$, and $C$ and $D$ have cubic or higher leading forms;

(3) $I=\left(L_{1} L_{2}+C, D, z^{2}\right)$ where $L_{1}, L_{2}, C$ and $D$ are as in (2).

Proof. We know that $I=H+A z^{2}$ where $H$ is an $m$-primary irreducible ideal of $A$. Also, we know that $H$ is generated by two parameters by Lemma 4.12 .

Suppose $H=\left(Q_{1}+C_{1}, Q_{2}+C_{2}\right)$ where $Q_{1}$ and $Q_{2}$ are quadratic forms and $C_{1}$ and $C_{2}$ are higher order terms. If $Q_{1}$ and $Q_{2}$ are relatively prime, then by considering the associated graded ring, we can see that everything of degree three or higher is contained in $H$. Thus $H=\left(Q_{1}, Q_{2}\right)$ and the third independent quadratic form will be the socle $\bmod H$.

If $Q_{1}$ and $Q_{2}$ are not relatively prime, we can write $H=\left(L L_{1}+C_{1}, L L_{2}+C_{2}\right)$. If $L$ and $L_{1}$ are independent over $K$, then they span the space of linear forms and we can write $L_{2}=a L+b L_{1}$. This implies that $L L_{2}=a L^{2}+b L L_{1}$. Hence we may rewrite $H$ as $\left(L L_{1}+C_{1}, L^{2}+C_{2}{ }^{\prime}\right)$. A similar argument applies if $L$ and $L_{2}$ are 
independent. If $L, L_{1}$ and $L_{2}$ are all dependent, then $H=\left(L^{2}+C_{1}, L^{2}+C_{2}\right)=$ $\left(L^{2}+C_{1}, C_{2}\right)$.

If $H=\left(L L_{1}+C_{1}, L^{2}+C_{2}{ }^{\prime}\right)$, since we must have $f \in H$, either $L L_{1}$ divides $f$ or $L^{2}$ divides $f$. Suppose $L$ does not divide $f$. Then the associated graded ring must contain everything of order three or higher and $f=\left(L+D_{2}\right)\left(L L_{1}+C_{1}\right)-$ $\left(L_{1}+D_{1}\right)\left(L^{2}+C_{2}^{\prime}\right)$. But everything on the right-hand side has order three or higher; hence $L$ divides $f$.

If $H=\left(L^{2}+C_{1}, C_{2}\right)$, then $L^{2}$ must divide $f$. To see this, note that if $C_{2}$ divides $f$, then $f$ will be a minimal generator of $H$. Since $z^{2} \in I$ and $z^{3}=-f$, if $f$ is a minimal generator of $H$, then $I$ will be generated by $z^{2}$ and the other minimal generator of $H, L^{2}+C_{1}$. In other words, $I$ will be generated by parameters and we know that the socle mod $I$ is contained in $I^{F}$ by Proposition 4.3.

(4.14) Comment. The remaining cases have proved to be very challenging. In particular, even the question of whether $x y z \in\left(x^{2}, y^{2}, z^{2}\right)^{*}$ is quite difficult. A. Singh has given an argument using determinants of matrices of binomial coefficients to show that indeed $x y z \in\left(x^{2}, y^{2}, z^{2}\right)^{*}$ for all $p$ and $x y z \in\left(x^{2}, y^{2}, z^{2}\right)^{F}$ for $p \equiv 2 \bmod 3$ [Si].

\section{Generalizations to Other Rings}

Many of the results in this paper can be generalized to rings of the form $K[[x, y, z]] /\left(z^{3}-F(x, y)\right)$ where $F(x, y)$ is a homogeneous polynomial of degree three, $K$ is a field of characteristic $p$ and $p \neq 3$. We first note that the maximal ideal, $m$, is the test ideal for these rings. For $p>3$ this is a consequence of a tight closure interpretation of the Kodaira Vanishing Theorem for Gorenstein rings in dimension two [HuS, (4.5) and (5.4)].

We give a proof for all positive prime characteristics here.

(5.1) Proposition. Let $R=K[[x, y, z]] /\left(z^{3}-F(x, y)\right)$, where $K$ is a field of characteristic $p$, and $F(x, y)$ is a homogeneous polynomial of degree three. Then $m$ is the test ideal for $R$.

Proof. The beginning of the proof is the same as the beginning of the proof of Proposition 1.4. We can show that it is sufficient to check that $\lambda_{3} x^{t-1} y^{t-1} z \notin\left(x^{t}, y^{t}\right)^{*}$ and $\lambda_{1} x^{t-2} y^{t-1} z^{2}+\lambda_{2} x^{t-1} y^{t-2} z^{2} \notin\left(x^{t}, y^{t}\right)^{*}$. The proof that $\lambda_{3} x^{t-1} y^{t-1} z \notin\left(x^{t}, y^{t}\right)^{*}$ is also the same as the proof in Proposition 1.4.

Suppose $\lambda_{1} x^{t-2} y^{t-1} z^{2}+\lambda_{2} x^{t-1} y^{t-2} z^{2} \in\left(x^{t}, y^{t}\right)^{*}$. This implies that $\lambda_{1} x z^{2}+$ $\lambda_{2} y z^{2} \in\left(x^{t}, y^{t}\right)^{*}: x^{t-2} y^{t-2}$. We know that $\left(x^{t}, y^{t}\right)^{*}: x^{t-2} y^{t-2} \subseteq\left(x^{2}, y^{2}\right)^{*}$ by the usual colon capturing argument [HH1, Theorem 7.15a]. If $\lambda_{1} x z^{2}+\lambda_{2} y z^{2} \in$ $\left(x^{2}, y^{2}\right)^{*}$, then we can find $c \neq 0$ such that $c\left(\lambda_{1} x+\lambda_{2} y\right)^{q} z^{2 q} \in\left(x^{2 q}, y^{2 q}\right)$ for all $q$. Write $2 q=3 h+2$. Using the basic relation in $R$, this implies that $c\left(\lambda_{1} x+\lambda_{2} y\right)^{q} F^{h} \in\left(x^{2 q}, y^{2 q}\right)$ or $c F^{h} \in\left(x^{2 q}, y^{2 q}\right):\left(\lambda_{1} x+\lambda_{2} y\right)^{q}$. This is equivalent to having $c F^{h} \in\left(x^{2 q}, y^{2 q}, x^{q} y^{q},\left(\lambda_{1} x-\lambda_{2} y\right)^{q}\right)=\left(x^{2 q},\left(\lambda_{1} x-\lambda_{2} y\right)^{q}\right)$. We can use $F$ as a test element and then $F^{h+1} \in\left(x^{2 q},\left(\lambda_{1} x-\lambda_{2} y\right)^{q}\right)$. Suppose $F^{h+1}=A x^{2 q}+$ $B\left(\lambda_{1} x-\lambda_{2} y\right)^{q}$, where $A, B \in K[[x, y]]$. By a degree argument we must have $F^{h+1}=$ $\left(a_{1} x+a_{2} y\right) x^{2 q}+B\left(\lambda_{1} x-\lambda_{2} y\right)^{q}$, where $a_{1}, a_{2} \in K$. Let $F_{x}$ denote the partial derivative of $F$ with respect to $x$. Taking derivatives twice yields $(h+1) h F^{h-1} F_{x}^{2}+$ $(h+1) F^{h} F_{x x}=B_{x x}\left(\lambda_{1} x-\lambda_{2} y\right)^{q}$. This implies that $(h+1) h F_{x}^{2}+(h+1) F F_{x x}=0$, because after we divide both sides by $F^{h-1}$, we still have a high power of $\left(\lambda_{1} x-\lambda_{2} y\right)$ that must divide the left-hand side. This implies that $h F_{x}^{2}+F F_{x x}=0$. We can 
assume that $F$ has distinct linear factors, and by making a change of variable if necessary, we can assume that $F=x y(a x+b y)$ with $a, b \neq 0$. Write $L$ for $(a x+b y)$. Then $F=x y L, F_{x}=y(a x+L)$ and $F_{x x}=2 a y$. Substituting yields $h y^{2}(a x+L)^{2}+x y L(2 a y)=0$ or $h y^{2}\left(a^{2} x^{2}+2 a x L+L^{2}\right)+2 a x y^{2} L=0$. If $p \neq 2$, then this implies that $L$ divides $h a^{2} x^{2} y^{2}$ which is not possible. If $p=2$, then we must have $h y^{2}(a x+L)^{2}=h y^{2}(b y)^{2}=0$. This implies that $h b^{2}=0$, but $h$ can be chosen larger than 2 and $b$ was assumed to be non-zero.

As $m$ is the test ideal, we know that if $u \in I^{*} \backslash I$, then $u$ is in the socle mod $I$ (Proposition 1.5). We will combine this fact with the following analogue of Proposition 4.3 in order to make the generalizations.

(5.2) Proposition. Let $R=K[[x, y, z]] /\left(z^{3}-F(x, y)\right)$, where $K$ is a field of characteristic $p, p \equiv 2 \bmod 3$, and $F(x, y)$ is a homogeneous polynomial of degree three. Let $I$ be an irreducible m-primary ideal of $R$ with $I \subseteq(x, y)$. Suppose u represents the socle mod $I$. Then $u \in I^{F}$.

Proof. We know that there is an injection $R /(x, y) \hookrightarrow R / I$ (Proposition 4.1). It suffices to see that $z^{2 p} \in\left(x^{p}, y^{p}\right)$. Suppose $p=3 h+2$. Then $z^{2 p}=F^{2 h+1} z$. Now it is enough to see that $F^{2 h+1} \in\left(x^{p}, y^{p}\right)$ in $K[[x, y]]$. The degree of $F^{2 h+1}$ is $2 p-1$, so every term of $F^{2 h+1}$ has a factor of $x^{p}$ or $y^{p}$. In other words, $F^{2 h+1} \in\left(x^{p}, y^{p}\right)$. Hence $u \in I^{F}$ (4.1).

The classification of irreducibles (Proposition 4.4) also follows essentially unchanged. Thus the irreducible $m$-primary ideals of $R=K[[x, y, z]] /\left(z^{3}-F(x, y)\right)$ are exactly the ideals of the form $(H, H, H),(H, H: F, H: F)$ and $(H, H, H: F)$ where $H$ is an irreducible $m$-primary ideal of $K[[x, y]]$ and $F=F(x, y)$. As before, $(H, H, H) \subseteq(x, y)$ and $(H, H: F, H: F)$ and $(H, H, H: F)$ are both contained in the ideal $(x, y)$ as long as $F \notin H$. We know then that $I^{\mathrm{F}}=I^{*}$ and $I \neq I^{*}$ in these cases. More generally, for any irreducible $m$-primary ideal of $R$ contained in $(x, y)$ we have that $I^{\mathrm{F}}=I^{*}$.

\section{REFERENCES}

[Ab] I. Aberbach, Tight closure in F-rational rings, Nagoya Math. J. 135 (1994), 43-54. MR 95g:13020

[E] D. Eisenbud, Commutative Algebra With a View Toward Algebraic Geometry, Graduate Text in Mathematics 150, Springer-Verlag, New York, 1995. MR 97a:13001

[Fi] N. J. Fine, Binomial coefficients modulo a prime, Amer. Math. Monthly, 54 (1947), 589592. MR 9:331b

[HH1] M. Hochster and C. Huneke, Tight closure, invariant theory, and the Briançon-Skoda theorem, J. Amer. Math. Soc. 3 (1) (1990), 31-116. MR 91g:13010

[HH2] M. Hochster and C. Huneke, Tight closure of parameter ideals and splitting in module-finite extensions, J. Algebraic Geometry 3 (1994), 599-670. MR 95k:13002

[HR] M. Hochster and J. L. Roberts, The purity of the Frobenius and local cohomology, Adv. Math. 21 (1976), 117-172. MR 54:5230

[Hu] C. Huneke, Tight closure and its applications, C.B.M.S. Regional Conf. Ser. in Math. No. 88 (1996). MR 96m:13001

[HuS] C. Huneke and K. E. Smith, Tight closure and the Kodaira vanishing theorem, J. Reine Angew. Math. 484 (1997), 127-152. MR 98e:13007

[K] E. Kunz, Characterizations of regular local rings of characteristic p, Amer. J. Math. 91 (1969), 772-784. MR 40:5609

[L] E. Lucas, Théorie des functions numérique simplement pèriodiques, Amer. J. Math. 1 (1878), 184-240.

[N] M. Nagata, Local Rings, Interscience, New York, 1962. MR 27:5790 
[Si] A. Singh, A computation of tight closure in diagonal hypersurfaces, Journal of Algebra 203 (1998), 579-589. CMP 98:12

[Sm1] K. E. Smith, Tight closure of parameter ideals, Invent. Math. 115 (1) (1994), 41-60. MR 94k:13006

[Sm2] K. E. Smith, Test ideals in local rings, Trans. Amer. Math. Soc. 347 (9) (1995), 3453-3472. MR 96c: 13008

[Sm3] K. E. Smith, Tight closure in graded rings, J. Math. Kyoto Univ. 37 (1) (1997), 35-53. MR 98e:13009

Mathematics and Computer Science Department, Gustavus Adolphus College, 800 W. College Avenue, St. Peter, Minnesota 56082-1498

E-mail address: mmcdermo@gac.edu 